\title{
Coherent Power Measurements with a Compact Airborne Ka-Band Precipitation Radar
}

\author{
ANDREW L. PAZMANY \\ ProSensing Inc., Amherst, Massachusetts \\ SAMUEL J. HAIMOV \\ University of Wyoming, Laramie, Wyoming
}

(Manuscript received 28 March 2017, in final form 2 November 2017)

\begin{abstract}
Coherent power is an alternative to the conventional noise-subtracted power technique for measuring weather radar signal power. The inherent noise-canceling feature of coherent power eliminates the need for estimating and subtracting the noise component, which is required when performing conventional signal power estimation at low signal-to-noise ratio. The coherent power technique is particularly useful when averaging a high number of samples to improve sensitivity to weak signals. In such cases, the signal power is small compared to the noise power and the required accuracy of the estimated noise power may be difficult to achieve. This paper compares conventional signal power estimation with the coherent power measurement technique by investigating bias, standard deviation, and probability of false alarm and detection rates as a function of signal-to-noise ratio and threshold level. This comparison is performed using analytical expressions, numerical simulations, and analysis of cloud and precipitation data collected with the airborne solid-state Ka-band precipitation radar (KPR) operated by the University of Wyoming.
\end{abstract}

\section{Introduction}

Radar reflectivity factor $Z$ is most commonly calculated from noise-subtracted signal power measurements (Ulaby et al. 1982, 492-495; Doviak and Zrnić 1993; Skolik 1990, 23.1-33; McDonough and Whalen 1995). At low signal-to-noise ratio (SNR), this technique requires an independent estimation of the noise component of the signal-plus-noise measurements. This is typically accomplished by shifting the measurement to a signal-free region in time (Ivić et al. 2013, 2014b) or frequency (Hildebrand and Sekhon 1974; Siggia and Passarelli 2004; Urkowitz and Nespor 1992). However, the noise component cannot always be estimated with sufficient accuracy to prevent significant additional errors in the signal estimate. For example, if the noise samples are gathered prior to each transmit pulse, then multitrip echoes can contaminate the noise estimate. Even in the absence of multitrip echo contamination, the transmitted pulse leakage can desensitize receiver

Corresponding author: Andrew L. Pazmany, pazmany@ prosensing.com amplifiers such that the receiver gain is a function of range. If the noise samples are collected offset in frequency, then the noise estimate may be biased as result of uncompensated variation in the receiver frequency response. The noise power also depends on the receiver noise figure and the radiometric brightness temperature of the scene, so measuring the noise power corresponding to an internal matched load or temporal averaging "noise" gates when the scene brightness temperature varies can lead to significant errors (Ivić et al. 2013, 2014b). A better method is to average the noise power in several range gates past the farthest scatterers. To ensure signal-free gates, the pulse repetition interval (PRI) needs to be increased beyond what would be necessary to sample the maximum signal range. However, this reduces the folding velocity and the number of samples available for signal measurement at a given temporal averaging interval, consequently, degrading sensitivity. Furthermore, it is often desirable to average many hundreds or even thousands of samples to boost sensitivity, thereby extending the signal power measurement range $10-20 \mathrm{~dB}$ below the receiver noise floor. As a result, a significant portion of the 
measurements are made well below unity SNR, where to avoid large errors in the estimated signal power, the required noise component measurement accuracy can be difficult to achieve. For example, when integrating 2000 pulses, signals that are $10 \mathrm{~dB}$ below the noise level can be detected with a high probability of detection $(>90 \%)$ at a false alarm rate of 0.01 . However, at this signal level, a \pm 0.25 - $\mathrm{dB}$ error in the estimated noise component introduces approximately +2 to $-4 \mathrm{~dB}$ error in the calculated signal power.

The coherent power (CP) technique is an alternative method for measuring the radar received signal power without the need for estimating the noise power. The key to this technique is correlating pairs of signal-plusnoise samples in which the signal components are correlated but the noise components are independent. This can be implemented with dual-polarized radars operating in simultaneous transmit and receive (STAR) mode (Seliga and Bringi 1976, Zrnić 1996) by computing the lag-0 cross-correlation magnitude, also known as copolar power (Keranen and Chandrasekar 2014), or using time-delayed $\mathrm{CP}$ by computing the lag- 1 autocorrelation magnitude. This time-delayed version of $\mathrm{CP}$, normalized to the signal power, has been used for censoring (Bell et al. 2013), and the combination of dual-polarization and the time-delayed version of $\mathrm{CP}$ has been shown to improve signal detection (Ivić et al. 2014a, 2009b).

The polarimetric method depends on the fact that the vertically and horizontally polarized copolar backscatter from precipitation is typically highly correlated, while the noise components of the vertically and horizontally polarized receiver channels are independent. The timedelayed version of the $\mathrm{CP}$ technique relies on the target fading much more slowly than the pulse-pair spacing, so the signal components of such closely spaced pulses are highly correlated but the noise components are not. The pulse-to-pulse phase difference of the signal components has a mean offset that depends on the mean Doppler velocity, but as long as the PRI is much shorter than the inverse of the spectrum width, this phase difference will be approximately constant. However, the noise component phase is uniformly distributed over $2 \pi$ from pulse to pulse; so, if the pulse-pair phasors (complex product of the sample voltage from pulse 2 and the complex conjugate of the sample voltage from pulse 1) are coherently averaged, then the noise component decreases while the magnitude of the signal component remains constant. This allows CP to estimate low SNR signal power without an independent estimate of the noise power.

Previously reported applications of $\mathrm{CP}$ have been used exclusively to improve signal detection or censoring (Ivić et al. 2009b; Bell et al. 2013; Ivić et al. 2014a; Keranen and Chandrasekar 2014). This paper proposes the use of CP as an alternative to the commonly used noise-subtracted power (NSP) technique for measuring signal power, particularly at low SNR, when the estimation and subtraction of the noise component is necessary with the NSP method. The CP technique is compared to the conventional NSP method when both techniques are properly implemented; that is, when the noise component estimate accuracy is much better than that of the signal-plus-noise measurements and when CP pulse-pair spacing is much smaller than the inverse of the signal bandwidth. The analysis presented in this paper shows that these techniques have similar potential accuracy and detection probabilities with some variation depending on the desired probability of false alarm. However, the important benefit of the $\mathrm{CP}$ technique is that it can maintain this benchmark accuracy at low SNR when the noise-subtracted power technique potentially degrades as a result of errors in the noise component estimate. This paper focuses on the timedelayed version of $\mathrm{CP}$, but some of the results can be applied to the dual-polarized CP method.

In section 2 the conventional NSP technique is reviewed. In section 3 , the $\mathrm{CP}$ estimate probability density function, bias, and standard deviation are characterized, and the probability of false alarm (PFA) and probability of detection (PD) of the CP and NSP techniques are compared for various threshold levels and SNRs. In section 4 a low SNR CP bias correction procedure is described, and in section 5 a correction for bias caused by finite pulse-topulse signal correlation is outlined. In section 6 analytical and simulation results are validated with cloud and precipitation data collected with the University of Wyoming (UWyo) airborne Ka-band precipitation radar (KPR).

\section{Noise-subtracted power}

The well-known NSP measurement technique (Ulaby et al. 1982, 492-493; Doviak and Zrnić 1993, p. 125 and p. 145) is reviewed first. The mean measured noise power $\left\langle\hat{P}_{n}\right\rangle$ from $N_{n}$ noise samples can be expressed in terms of the standard deviation $\left(\sigma_{n}\right)$ of the zero-mean Gaussian-distributed (Goodman 1975) $I$ and $Q$ noise samples as

$$
\left\langle\hat{P}_{n}\right\rangle=\left\langle\frac{1}{N_{n}} \sum_{k=1}^{N_{n}}\left|I_{n}(k)+j Q_{n}(k)\right|^{2}\right\rangle=2 \sigma_{n}^{2}=P_{n} .
$$

Similarly, the mean of $N$ signal-plus-noise power samples is

$$
\begin{aligned}
\left\langle\hat{P}_{s+n}\right\rangle & =\left\langle\frac{1}{N} \sum_{k=1}^{N}\left|I_{s}(k)+j Q_{s}(k)+I_{n}(k)+j Q_{n}(k)\right|^{2}\right\rangle, \\
& =P_{s}+P_{n} .
\end{aligned}
$$


The instantaneous power samples (i.e., power samples from a single transmitted pulse) are random variables with exponential distribution with equal mean and standard deviation (Ulaby et al. 1982, p. 481). The probability density function (pdf) of the average of $N_{I}$ independent signal-plus-noise power samples is a chi-squared distribution of $2 N_{I}$ degrees of freedom (Ulaby et al. 1982, 486487; Mead 2016; Ivić et al. 2009b, appendix A)

After noise subtraction, the signal estimate variance is the sum of the signal-plus-noise measurement and noise component estimate variances. If the number of noiseonly samples, $N_{n}$, is much higher than $N_{I}$, then the standard deviation of the noise-subtracted power estimate can be approximated as

$$
\sigma_{\hat{P}_{s}}=\sqrt{\frac{\left(P_{s}+P_{n}\right)^{2}}{N_{I}}+\frac{P_{n}^{2}}{N_{n}}} \approx \frac{P_{s}+P_{n}}{\sqrt{N_{I}}} .
$$

This approximation assumes error-free noise estimation, requiring that $N_{n} \gg N_{I}$ and that the mean noise power in the noise sampling region equals the noise component of the signal-plus-noise measurement.

The term $N_{I}$ is the equivalent number of independent signal-plus-noise samples (Doviak and Zrnić 1993, 127129), not necessarily the total number of samples averaged $(N)$. At very low SNR, $N_{I}$ can be assumed to equal $N$, because the receiver noise is usually uncorrelated from sample to sample, even at very high radar pulse repetition frequency (PRF). ${ }^{1}$ However, at high SNR, $N_{I}$ approaches the number of equivalent independent signal samples, which can be just a fraction of $N$ when the PRF is high relative to the signal spectrum width; that is, the normalized spectrum width $\ll 1$. Normalized spectrum width is defined as $\sigma_{v n}=2 \sigma_{v} T_{s} / \lambda$ (Doviak and Zrnić 1993, p. 128), where $\sigma_{v}$ is the Doppler velocity spectrum standard deviation, $T_{s}$ is the sampling time interval, and $\lambda$ is the radar wavelength. The change in the number of independent samples versus the total number of samples $\left(N_{I} / N\right)$ as a function of SNR and $\sigma_{v n}$, for Gaussian signal power spectrum and square law detection, is illustrated in Fig. 1. The curves were calculated by combining Eqs. (6.4), (6.11a), and (6.12) in Doviak and Zrnić (1993):

$$
\begin{aligned}
\rho_{S}\left(m T_{S}\right) & =\left[\frac{\left|R\left(m T_{s}\right)\right|}{R(0)}\right]^{2}, \\
& =\left\{\frac{\mathrm{SNR} \exp \left[-2\left(\pi m \sigma_{v n}\right)\right]+\delta_{m}}{\mathrm{SNR}+1}\right\}^{2}, \text { and }
\end{aligned}
$$

\footnotetext{
${ }^{1}$ Noise samples collected from successive range gates can be partially correlated when the data system sampling frequency is faster than the effective receiver (noise) bandwidth.
}

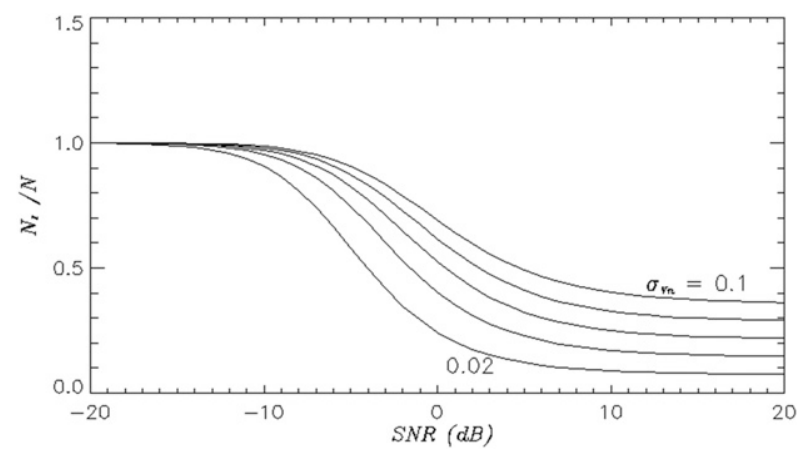

FIG. 1. Simulated ratio of the number of independent samples $\left(N_{I}\right)$ and the total number of samples averaged $(N)$ as a function of SNR and for normalized signal spectrum width $\left(\sigma_{v n}\right)$ of $0.02,0.04,0.06$, 0.08 , and 0.1 . The high SNR limit of $N_{I}$ according to Doviak and Zrnić $(1993,127-128)$ is indicated with thicker red line ends in the plot.

$$
\frac{N_{I}}{N}=\left[\sum_{m=-(N-1)}^{N-1} \frac{N-|m|}{N} \rho_{S}\left(m T_{S}\right)\right]^{-1},
$$

where $\rho_{s}$ is the square law detector output normalized correlation, $R$ is the signal-plus-noise autocorrelation, and $\delta_{m}=1$ when $m=0$ and 0 otherwise. For $N>1 / \sigma_{v n}$ and $\sigma_{v n} \ll 1$,

$$
\frac{N_{I}}{N} \approx(\mathrm{SNR}+1)^{2}\left(\frac{\mathrm{SNR}^{2}}{2 \sigma_{v n} \sqrt{\pi}}+2 \mathrm{SNR}+1\right)^{-1} .
$$

The probability of detection of noise-subtracted power $\left(\mathrm{PD}_{p}\right)$ for a given threshold $(T)$ can be expressed using the cumulative distribution function (CDF) for a chi-squared distribution with $2 N_{I}$ degrees of freedom [Abramowitz and Stegun 1964, their Eq. (26.4.1); cf. Wolfram 2012a] denoted by $D$ [Mead 2016, rearranged form of Eq. (40)]:

$$
\mathrm{PD}_{p}=1-D_{2 N_{I}}\left(2 N_{I} \frac{\frac{T}{P_{n}}+1}{\mathrm{SNR}+1}\right)
$$

We can normalize the threshold to the effective noise floor $\left(P_{n} / \sqrt{N_{I}}\right)$ after noise subtraction by introducing a threshold factor, $T_{f}$, such that $T_{f}=\left(T \sqrt{N_{I}} / P_{n}\right)$, to obtain the following expression for the detection rate:

$$
\mathrm{PD}_{p}=1-D_{2 N_{I}}\left(2 N_{I} \frac{\frac{T_{f}}{\sqrt{N_{I}}}+1}{\mathrm{SNR}+1}\right) .
$$

Equation (8) gives the probability of detection for the average of $N_{I}$ independent signal-plus-noise samples 


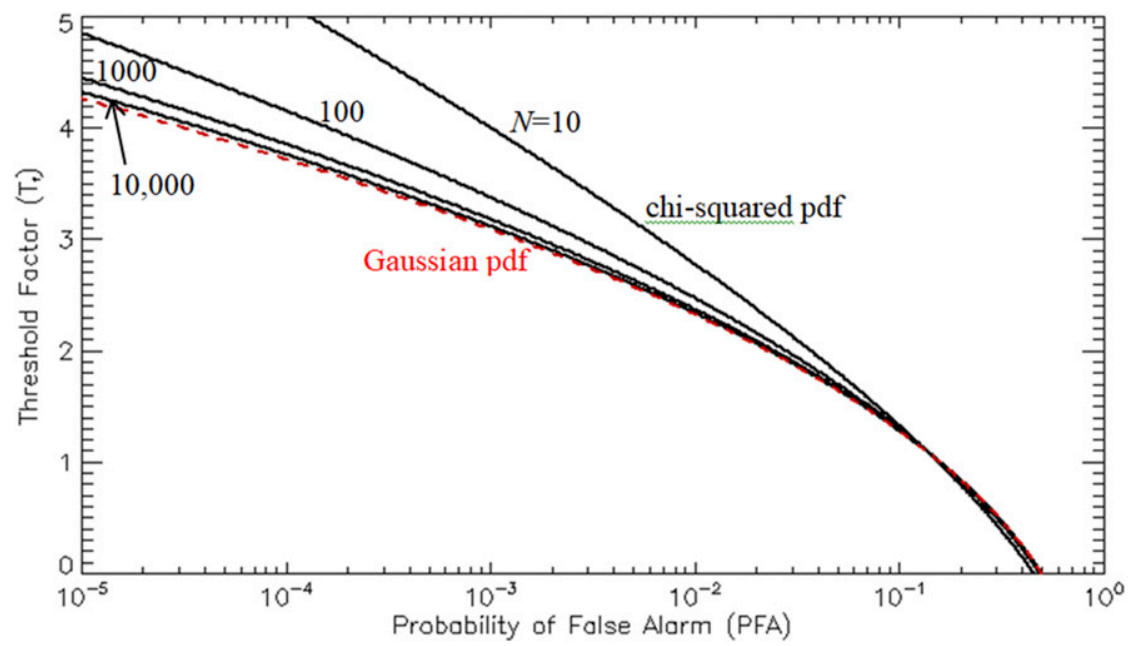

FIG. 2. NSP technique probability of false alarm vs thresholding evaluated using the chisquared pdf (black lines) and based on the Gaussian pdf approximation (red dashed line), both with perfect noise subtraction. Chi-squared distribution is slow to converge to Gaussian, so the approximations should be used only for large $N$ or high PFA rates.

after perfect noise subtraction $\left(N_{n} \gg N\right.$, and the noise component estimate is unbiased). The chi-squared distribution converges to the Gaussian distribution with an increasing number of independent samples averaged; so, for large $N_{I}$ the probability of detection can be approximated using the noise-subtracted mean and standard deviation from Eqs. (2) and (3) as

$\mathrm{PD}_{p} \cong \frac{1}{\frac{P_{n}+P_{s}}{\sqrt{N_{I}}} \sqrt{2 \pi}} \int_{T}^{\infty} e^{-\frac{\left(x-P_{s}\right)^{2}}{2 \frac{\left(P_{n}+P_{s}\right)^{2}}{N_{I}}}} d x$ for large $N_{I}$.

$\mathrm{PD}_{p} \cong \frac{1}{2}\left[1-\operatorname{erf}\left(\sqrt{\frac{1}{2}} \frac{T_{f}-\mathrm{SNR} \sqrt{N_{I}}}{1+\mathrm{SNR}}\right)\right]$ for large $N_{I}$,

where $\operatorname{erf}(x)=(2 / \sqrt{\pi}) \int_{0}^{x} e^{-t^{2}} d t$.

In the absence of any signal ( $\mathrm{SNR}=0)$, the probability of detection is the probability of false alarm (PFA):

$$
\begin{aligned}
& \mathrm{PFA}_{p}=1-D_{2 N_{I}}\left[2\left(T_{f} \sqrt{N}+N\right)\right], \\
& \mathrm{PFA}_{p} \cong \frac{1}{2}\left[1-\operatorname{erf}\left(\frac{T_{f}}{\sqrt{2}}\right)\right] \text { for large } N .
\end{aligned}
$$

A more useful form of these equations is the inverse, estimating the required threshold factor for a desired false alarm rate:

$$
T_{f}=\frac{D_{2 N_{I}}^{-1}\left(1-\mathrm{PFA}_{P}\right)-2 N}{2 \sqrt{N}} \text { and }
$$

$$
T_{f} \cong \sqrt{2} \operatorname{erf}^{-1}\left(1-2 \mathrm{PFA}_{P}\right) \quad \text { for } \text { large } N
$$

A more detailed analysis of estimating the required threshold level to obtain a desired PFA was published by Ivić (2009a).

The Gaussian approximation might be easier to use than the inverse $D$ function, but chi-squared distribution is slow to converge to Gaussian; therefore, Eq. (14) should be used only if a large number of samples are averaged or for calculating thresholds that correspond to relatively high false alarm rates. The relationship between $T_{f}$ and PFA and the differences based on the use of the chi-squared and Gaussian pdfs are illustrated in Fig. 2. The Gaussian pdf approximation is shown as a red dashed line and the black lines are for the chi-squared pdf, both with perfect noise subtraction. The Gaussian approximation underestimates the required threshold level and results in a higher-than-desired PFA.

\section{Coherent power}

Similar to Eq. (1), a complex voltage sample can be expressed using zero-mean Gaussian $I$ and $Q$ signal and noise components as

$$
V(k)=I_{s}(k)+j Q_{s}(k)+I_{n}(k)+j Q_{n}(k) .
$$

The time-delayed CP estimate $\left(\hat{P}_{\mathrm{cp}}\right)$ is the magnitude of the autocorrelation function at $\operatorname{lag} m T_{s}$ :

$$
\hat{P}_{\mathrm{cp}}=\left|\frac{1}{N} \sum_{k=1}^{N} V^{*}(k) V(k+m)\right|,
$$




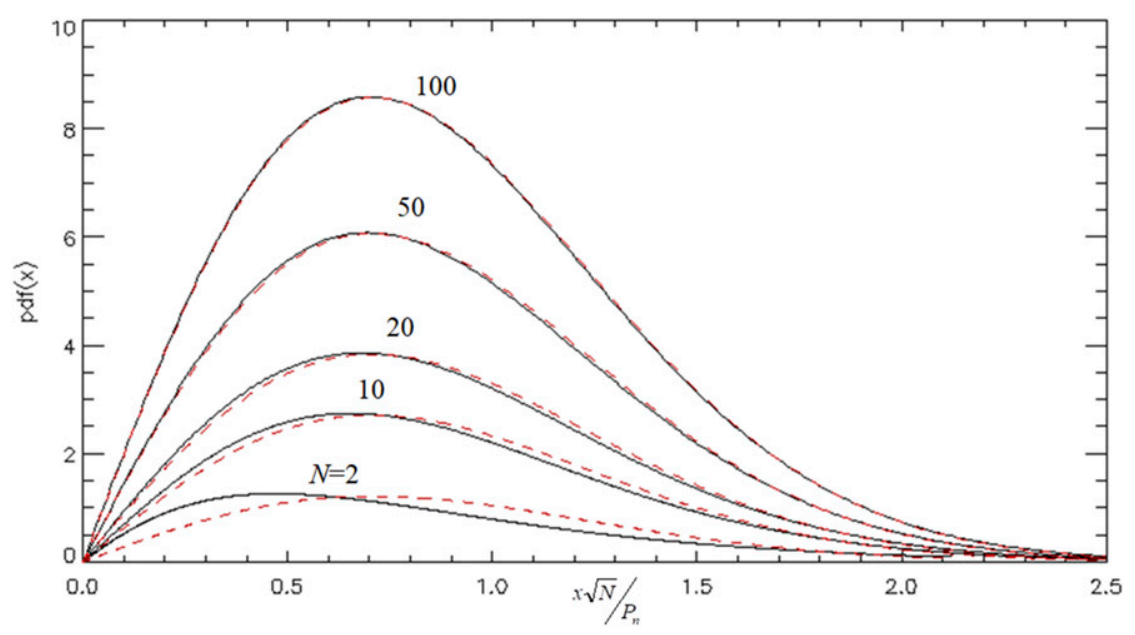

FIG. 3. CP estimate $\left(\widehat{P}_{\mathrm{cp}}\right)$ pdf for $N$ samples of noise (SNR $=0$ ). Equation (19) from Lee et al. (1994) (solid black lines), and Rayleigh approximation (dashed red lines).

where $m \ll N$, the superscript asterisk (*) denotes a complex conjugate, and the circumflex $\left(^{\wedge}\right)$ denotes the estimated value. An integer $m$ indicates uniform PRI, while staggered PRI would have a noninteger $m$.

\section{a. Noise-only coherent power}

In the absence of any signal ( $\mathrm{SNR}=0$ ), the noise-only $\hat{P}_{\text {cp }}$, in terms of Eqs. (15) and (16), can be written as

$$
\begin{aligned}
\hat{P}_{\mathrm{cp}}= & \mid \frac{1}{N} \sum_{k=1}^{N}\left[I_{n}(k) I_{n}(k+m)+Q_{n}(k) Q_{n}(k+m)\right. \\
& \left.+j I_{n}(k) Q_{n}(k+m)-j I_{n}(k+m) Q_{n}(k)\right] \mid .
\end{aligned}
$$

The $I_{n}$ and $Q_{n}$ noise samples are independent, zero-mean Gaussian random variables with a standard deviation $\sigma_{n}$. The products in Eq. (17) have a pdf of the modified Bessel function of the second kind (Wolfram 2012b) with a standard deviation $\sigma_{n}^{2}$. With increasing $N$, based on the central limit theorem (Papoulis 1984, p. 194), the real and imaginary components of the average in Eq. (17) converge toward independent Gaussian random variables, with zero mean and $\sqrt{2 / N}\left(\sigma_{n}^{2}\right)$ standard deviation. Coherent power is the magnitude of this phasor and the associated pdf converges to Rayleigh (Beckmann 1962; Papoulis 1984, p.138; Fortune 2005, p.18):

$\operatorname{pdf}_{\hat{P}_{\mathrm{cp}}}(x \mid$ noise $) \cong \frac{2 x N}{P_{n}^{2}} \exp \left(\frac{-N x^{2}}{P_{n}^{2}}\right)$ for large $N$.

The general expression for the noise-only $(\mathrm{SNR}=0) \mathrm{CP}$ pdf, which is also valid for small $N$, is Eq. (32) in Lee et al. (1994):

$$
\operatorname{pdf}_{\hat{P}_{\mathrm{cp}}}(x \mid \text { noise })=\frac{4 N^{N+1}}{\Gamma(N) P_{n}^{N+1}} x^{N} K_{N-1}\left(\frac{2 N x}{P_{n}}\right),
$$

where the symbol $\Gamma$ is the gamma function and $K_{N-1}$ is the modified Bessel function of the second kind. With dual-polarized CP, $P_{n}$ can be replaced with the geometric mean of the vertical $(\mathrm{V})$ - and horizontal $(\mathrm{H})$-polarized receiver noise floors $\left(P_{\mathrm{nv}}\right.$ and $P_{\mathrm{nh}}$, respectively) as $P_{n} \Rightarrow \sqrt{P_{\mathrm{nv}} P_{\mathrm{nh}}}$. The convergence of Eq. (19) toward the Rayleigh pdf [Eq. (18)] is fast with an increasing number of samples $(N)$, as illustrated in Fig. 3 . Furthermore, the Rayleigh pdf is easier and more efficient to use than Eq. (19) for the evaluation of $\hat{P}_{\mathrm{cp}}$ noise floor mean, variance, and thresholding required to achieve a desired false alarm rate, especially given the complexities of accurately evaluating $K_{N-1}$ in Eq. (19) for large $N$ (Jentschura and Lötstedt 2011).

Using the Rayleigh pdf approximation, the mean and standard deviation of the noise-only $\hat{P}_{\mathrm{cp}}$ are

$$
\left\langle\hat{P}_{\mathrm{cp}}\right\rangle \cong \frac{\sqrt{\pi}}{2} \frac{P_{n}}{\sqrt{N}} \text { for large } N
$$

and

$\sqrt{\left\langle\hat{P}_{\mathrm{cp}}^{2}\right\rangle-\left\langle\hat{P}_{\mathrm{cp}}\right\rangle^{2}} \cong \frac{\sqrt{4-\pi}}{2} \frac{P_{n}}{\sqrt{N}}$ for large $N$.

This Rayleigh approximation for $N=10$ and $N=100$ overestimates the mean by about $1 \%(0.04 \mathrm{~dB})$ and $0.1 \%(0.004 \mathrm{~dB})$ and underestimates the standard deviation by $5 \%$ and $0.5 \%$, respectively.

The CP probability of false alarm ( $\mathrm{PFA}_{\mathrm{cp}}$ ) can be approximated using the Rayleigh cumulative distribution function, $C(T), C(T)=1-e^{-\frac{T^{2}}{\left(2 s^{2}\right)}}$, where $s=P_{n} / \sqrt{2 N}$ as 


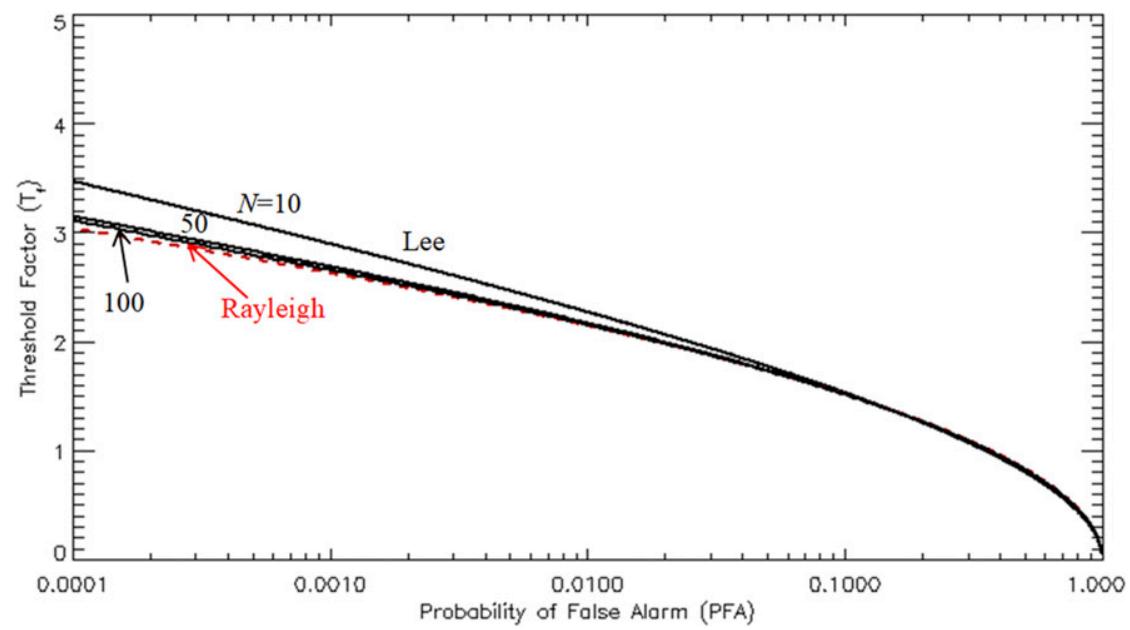

FIG. 4. CP probability of false alarm ( $\left.\mathrm{PFA}_{\mathrm{cp}}\right)$ vs threshold factor $\left(T_{f}\right)$ for $N=10,50$, and 100 samples averaged. Values calculated using the Lee et al. (1994) pdf of Eq. (19) (solid black lines) and those based on the Rayleigh approximation (dashed red line).

$\mathrm{PFA}_{\mathrm{cp}} \cong 1-C(T)=e^{-\frac{N T^{2}}{P_{n}^{2}}}=e^{-T_{f}^{2}}$ for large $N$.

The inverse form for estimating the required threshold factor for a desired false alarm rate is

$$
T_{f} \cong \sqrt{-\ln \left(\mathrm{PFA}_{\mathrm{cp}}\right)} \text { for large } N .
$$

A threshold level calculated using Eq. (23), based on Rayleigh pdf approximation, results in higher false alarm rates than using the Lee pdf [Eq. (19)], as shown in Fig. 4. With $N=10$ samples and a $10 \%$ intended false alarm rate, the threshold calculated with Eq. (23) yields $\sim 10.03 \% \mathrm{PFA}_{\mathrm{cp}}$; $N=50$ samples and $1 \%$ intended $\mathrm{PFA}_{\mathrm{cp}}$ yields $\sim 1.1 \%$; and $N=100$ samples and $0.1 \% \mathrm{PFA}_{\mathrm{cp}}$ yields $0.106 \%$.

\section{b. Signal-plus-noise coherent power}

In the presence of signal, $\hat{P}_{\mathrm{cp}}$, in terms of Eqs. (15) and (16), can be written as

$$
\hat{P}_{\mathrm{cp}}=\left|\frac{1}{N} \sum_{k=1}^{N}\left[I_{s}(k)+j Q_{s}(k)+I_{n}(k)+j Q_{n}(k)\right]^{*}\left[I_{s}(k+1)+j Q_{s}(k+1)+I_{n}(k+1)+j Q_{n}(k+1)\right]\right| .
$$

In the limit of large $N, \hat{P}_{\text {cp }}$ converges to the pulse-topulse correlated portion of the signal power:

$$
\begin{aligned}
\lim _{N \rightarrow \infty} \hat{P}_{\mathrm{cp}} & =\left|\left\langle V^{*}(k) V(k+m)\right\rangle\right|, \\
& =\rho\left(m T_{s}\right) P_{s}=\rho_{m} P_{s},
\end{aligned}
$$

where $\rho_{m}$ is the magnitude of the normalized signal correlation of weather targets, defined by Doviak and Zrnić (1993, p. 125).

For finite $N$, the multiplication in Eq. (24) can be carried out and the real and imaginary components combined as follows:

$$
\hat{P}_{\mathrm{cp}}=\left|\frac{1}{N} \sum_{k=1}^{N} V^{*}(k) V(k+m)\right|=\left|e^{j \varphi_{D}}\left(I_{\mathrm{cp}}+j Q_{\mathrm{cp}}\right)\right|,
$$

where $\varphi_{D}$ is the phase shift (radians) associated with the mean Doppler velocity $\left(\hat{v}_{D}\right)$ as $\varphi_{D}=4 \pi \hat{v}_{D} m T_{s} / \lambda$. Note that the phase shift as result of the mean Doppler velocity has been separated from $I_{\mathrm{cp}}$ and $Q_{\mathrm{cp}}$ so that the mean value of $Q_{\mathrm{cp}}$ is zero (see the appendix). Terms $I_{\mathrm{cp}}$ and $Q_{\mathrm{cp}}$ are independent random variables with the following mean and variance (see the appendix for details):

$$
\begin{aligned}
\left\langle I_{\mathrm{cp}}\right\rangle= & \alpha=\rho_{m} P_{s}, \\
\left\langle Q_{\mathrm{cp}}\right\rangle= & 0, \\
\operatorname{var}\left(I_{\mathrm{cp}}\right)= & \left\langle I_{\mathrm{cp}}^{2}\right\rangle-\left\langle I_{\mathrm{cp}}\right\rangle^{2}=s_{1}=\frac{\rho_{m}^{2} P_{s}^{2}}{N_{I}} \\
& +\frac{\left(1-\rho_{m}^{2}\right) P_{s}^{2}+2 P_{s} P_{n}+P_{n}^{2}}{2 N}, \quad \text { and } \\
\operatorname{var}\left(Q_{\mathrm{cp}}\right)= & \left\langle Q_{\mathrm{cp}}^{2}\right\rangle-\left\langle Q_{\mathrm{cp}}\right\rangle^{2} \\
= & s_{2}=\frac{\left(1-\rho_{m}^{2}\right) P_{s}^{2}+2 P_{s} P_{n}+P_{n}^{2}}{2 N} .
\end{aligned}
$$




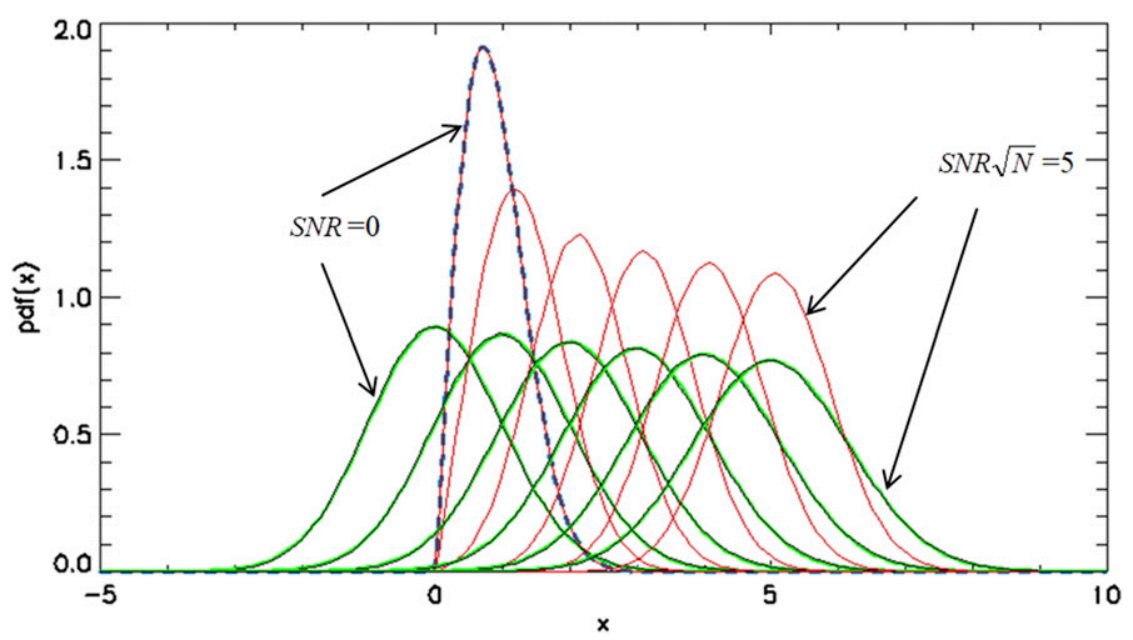

FIG. 5. Simulated CP (red) and NSP (black) estimate pdf for $\operatorname{SNR} \sqrt{N}=0-5$ and $N=1000$. Gaussian distribution thick green lines) and Rayleigh pdf (thick dashed blue line) are shown to illustrate that the CP pdf can be approximated as Rayleigh for SNR $=0$ and large $N$. CP estimate also converges toward the chi-squared (Gaussian for large $N$ ) power estimate pdf with increasing SNR and near-unity $\rho_{m}$.

At the limit of increasing SNR $(\mathrm{SNR} \rightarrow \infty)$ and unit $\rho_{m}$ $\left(\rho_{m} \rightarrow 1\right)$, the $\mathrm{CP}$ estimate converges to the signal power estimate $\left(\alpha \cong P_{s}, s_{1} \cong\left(P_{s}^{2} / N_{I}\right)\right.$ and $\left.s_{2} \cong 0\right)$ :

$$
\lim _{\substack{\mathrm{SNR} \rightarrow \infty \\ \rho_{m} \rightarrow 1}} \hat{P}_{\mathrm{cp}}=\hat{P}_{s}
$$

Therefore, just like the mean power estimate, the pdf of the CP estimate at high SNR and near-unity $\rho_{m}$ can be approximated with the chi-squared distribution. The shifting CP estimate density function from the Lee pdf of Eq. (19) at SNR $=0$, toward chi-squared at high SNR and unit $\rho_{m}\left(\rho_{m} \cong 1\right)$, is illustrated in Fig. 5 using simulations $(N=1000)$. The Rayleigh approximation of the zero SNR CP pdf and the Gaussian pdfs for increasing $\operatorname{SNR} \sqrt{N}$ are also plotted as a comparison.
Using the definition of $\mathrm{CP}$ and taking advantage of the independence of $I_{\mathrm{cp}}$ and $Q_{\mathrm{cp}}$, CP variance $\left(\sigma_{\mathrm{cp}}^{2}\right)$ can be simply related to the $\mathrm{CP}$ mean $\left(\mu_{\mathrm{cp}}\right)$ as (see also Beckmann 1962)

$$
\begin{aligned}
\sigma_{\mathrm{cp}}^{2} & =\left\langle\hat{P}_{\mathrm{cp}}^{2}\right\rangle-\left\langle\hat{P}_{\mathrm{cp}}\right\rangle^{2}=\left\langle\left(I_{\mathrm{cp}}+j Q_{\mathrm{cp}}\right)\left(I_{\mathrm{cp}}+j Q_{\mathrm{cp}}\right)^{*}\right\rangle-\left\langle\hat{P}_{\mathrm{cp}}\right\rangle^{2} \\
& =s_{1}+s_{2}+\alpha^{2}-\mu_{\mathrm{cp}}^{2}
\end{aligned}
$$

Unfortunately, there is no such simple exact expression for the mean.

For large $N$ and $N_{I}$, again based on the central limit theorem, $I_{\mathrm{cp}}$ and $Q_{\mathrm{cp}}$ converge toward Gaussian pdf and the CP estimate toward the Beckmann distribution, with the pdf given by Eq. (17) in Beckmann (1962):

$$
\operatorname{pdf}_{\hat{P}_{\mathrm{cp}}}(x) \cong \frac{x}{\sqrt{s_{1} s_{2}}} \exp \left(-\frac{\alpha^{2}}{2 s_{1}}-\frac{s_{2}-s_{1}}{4 s_{1} s_{2}} x^{2}\right) \times \sum_{k=0}^{\infty}(-1)^{k} \varepsilon_{k} I_{k}\left(\frac{s_{2}-s_{1}}{4 s_{1} s_{2}} x^{2}\right) I_{2 k}\left(\frac{\alpha}{s_{1}} x\right), \text { for large } N \text { and } N_{1}
$$

where $\varepsilon_{k}=1$ for $k=0$, and $\varepsilon_{k}=2$ for $k>0$ and $I_{k}(x)$ is a modified Bessel function of the first kind.

Note that for $k=0, I_{k}(0)=1$, and for $k>0, I_{k}(0)=0$, so the Beckmann distribution [Eq. (33)] reduces to Rayleigh [Eq. (18)] when $\rho_{m} P_{s}$ equals zero $(\alpha=0$ and $\left.s_{1}=s_{2}=P_{n}^{2} / 2 N\right)$ and converges to Gaussian for large $\alpha / \sqrt{s_{1}+s_{2}}$ [see the appendix of Beckmann (1962)]. At low SNR and near-unity $\rho_{m}\left[\left(\alpha / \sqrt{s_{1}+s_{2}}\right)<3\right]$, the first five terms $(k=0$ to 4$)$ in the infinite sum of Eq. (33) is sufficient to limit errors in the calculation of the first two moments to $<1 \%$ (for $N$ and $N_{I}>50$ ). For $\left(\alpha / \sqrt{s_{1}+s_{2}}\right)>3$ and $\left(s_{2} / s_{1}\right)<5$, the pdf can be approximated with Gaussian [see the appendix of Beckmann (1962)] and limit the first two moment errors to less than $2 \%$. 
Since the Beckmann distribution is valid only for large $N$ and numeric evaluation of the pdf, Eq. (33), and its moments is difficult, especially for large arguments, simulations were used to find a practical equation for the mean of the $\mathrm{CP}$ estimate in the transition region: SNR $>0$, finite $N$, and the partially correlated signal component $\left(0<\rho_{m}<1\right)$.

To develop an empirical formula for the mean $\mathrm{CP}$ estimate, only independent samples were considered in the simulation such that $\rho\left(T_{S}\right)=0$ and $N=N_{I}$. The signal terms in Eqs. (15) and (16) were decomposed to coherent and noncoherent signal components such that

$$
\begin{aligned}
V(k)= & I_{\mathrm{sc}}(k)+j Q_{\mathrm{sc}}(k)+I_{\mathrm{si}}(k)+j Q_{\mathrm{si}}(k)+I_{n}(k) \\
& +j Q_{n}(k), \quad \text { and } \\
V(k+m)= & I_{\mathrm{sc}}(k)+j Q_{\mathrm{sc}}(k)+I_{\mathrm{si}}(k+m)+j Q_{\mathrm{si}}(k+m) \\
& +I_{n}(k+m)+j Q_{n}(k+m),
\end{aligned}
$$

where $I_{\mathrm{sc}}$ and $Q_{\mathrm{sc}}$ are the correlated components and $I_{\mathrm{si}}$ and $Q_{\text {si }}$ are the independent components of $\left[V^{*}(k) V(k+m)\right]$, and all the $I$ and $Q$ terms are independent Gaussian random variables with variances $\sigma_{n}^{2}=1 / 2 P_{n}$ from Eq. (1), $\sigma_{\mathrm{sc}}^{2}=$ $\rho_{m} \mathrm{SNR} \sigma_{n}^{2}$, and $\sigma_{\mathrm{si}}^{2}=\left(1-\rho_{m}\right) \mathrm{SNR} \sigma_{n}^{2}$. The decomposition of the $I$ and $Q$ terms is allowed here because the sum of Gaussians is also a Gaussian random variable. It is also convenient for the modeling of a desired pulse-pair correlation; note that the same randomly generated numbers are used in both the first and second correlated terms $\left(I_{\mathrm{cs}}\right.$ and $\left.Q_{\mathrm{cs}}\right)$ of the pulse-pairs, so $\left\langle V^{*}(k) V(k+m)\right\rangle=$

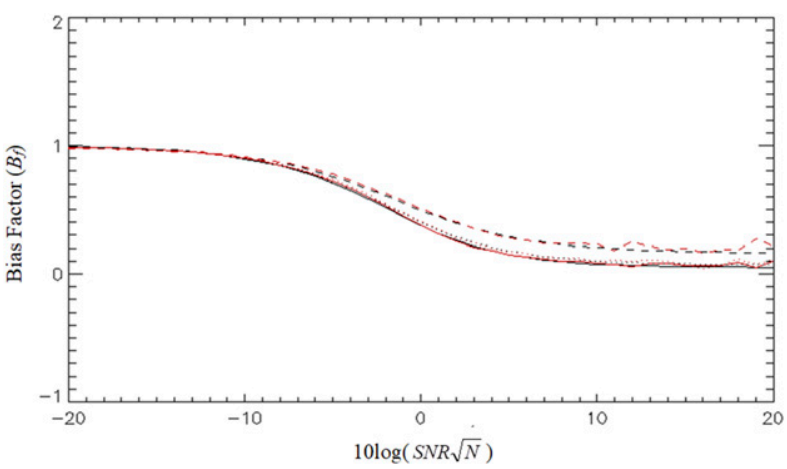

FIG. 6. CP bias factor as a function of coherent signal component-to-noise ratio and number of samples averaged. Simulation results (red) and Eq. (39) approximation (black); $N=100$ (solid line), $N=50$ (dots), and $N=10$ (dashes).

$\left\langle\left[I_{\mathrm{cs}}(k)+j Q_{\mathrm{cs}}(k)\right]^{*}\left[I_{\mathrm{cs}}(k)+j Q_{\mathrm{cs}}(k)\right]\right\rangle=\rho_{m} P_{s}$. Since pulsepair processing preserves only lag- 0 (power) and lag- $m T_{s}$ correlations, while all other signal spectrum shapes are lost, this simple model is adequate to simulate independent weather radar pulse-pairs samples.

The noncoherent signal and noise terms can be further combined as independent zero-mean Gaussian $\tilde{I}(k)$ and $\tilde{Q}(k)$ terms with variance $\left[\left(1-\rho_{m}\right) \mathrm{SNR}+1\right] \sigma_{n}^{2}$, to express the signal-plus-noise voltage sample as

$$
V(k)=I_{\mathrm{cs}}(k)+j Q_{\mathrm{cs}}(k)+\tilde{I}_{n}(k)+j \tilde{Q}_{n}(k),
$$

and the corresponding $\mathrm{CP}$ estimate as

$$
\hat{P}_{\mathrm{cp}}=\left|\frac{1}{N} \sum_{k=1}^{N}\left[I_{c s}(k)+j Q_{\mathrm{cs}}(k)+\tilde{I}_{n}(k)+j \tilde{Q}_{n}(k)\right]^{*}\left[I_{\mathrm{cs}}(k)+j Q_{\mathrm{cs}}(k)+\tilde{I}_{n}(k+m)+j \tilde{Q}_{n}(k+m)\right]\right|
$$

To characterize the diminishing $\mathrm{CP}$ bias with increasing $\operatorname{SNR} \rho_{m} /\left[\operatorname{SNR}\left(1-\rho_{m}\right)+1\right]$, we introduce the bias factor $\left(B_{f}\right)$ as

$$
B_{f}=2 \sqrt{\frac{N}{\pi}} \frac{\left\langle\hat{P}_{\mathrm{cp}}\right\rangle-\rho_{m} P_{s}}{\left(1-\rho_{m}\right) P_{s}+P_{n}} .
$$

The bias factor $B_{f}$ relates the receiver noise $P_{n}$ plus the noiselike uncorrelated portion of the pulse-pair sample, $\left(1-\rho_{m}\right) P_{s}$, to the CP bias: $\left\langle\hat{P}_{\mathrm{cp}}\right\rangle-\rho_{m} P_{s}$. At zero SNR and large $N, B_{f}=1$ according to Eq. (20), while with increasing SNR and $\rho_{m}$ approaching $1, B_{f}$ converges to zero. In the transition region, $B_{f}$ was estimated from simulations for $N=10,50$, and 100 as a function of $\mathrm{SNR}_{c} \sqrt{N}$ (red lines), as shown in Fig. 6, along with the approximated expression for $B_{f}$ (black lines):

$$
B_{f} \approx \frac{1}{2 \sqrt{N}}+\left(1-\frac{1}{2 \sqrt{N}}\right)\left\{\left[\left(2 \sqrt{\frac{N}{\pi}} \mathrm{SNR}_{c}\right)^{2.25-\frac{1}{\sqrt{N}}}+1\right]^{\frac{1}{2.25-\frac{1}{\sqrt{N}}}}-2 \sqrt{\frac{N}{\pi}} \mathrm{SNR}_{c}\right\}
$$




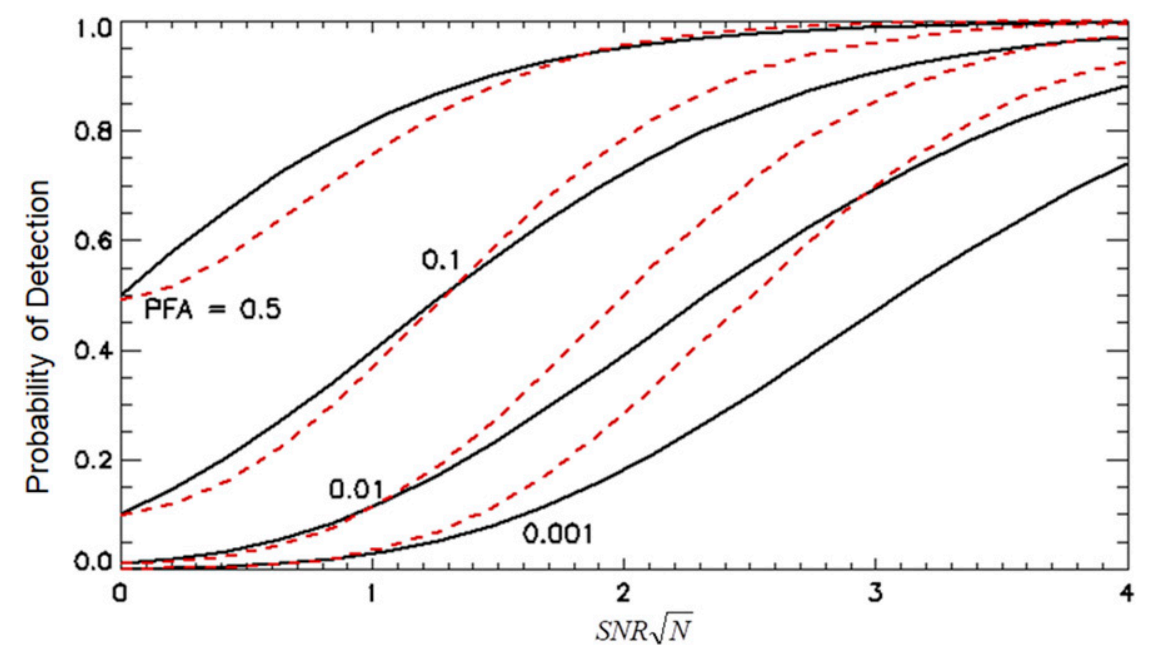

FIG. 7. NSP (solid black) and CP technique (dashed red) PD as a function of SNR $\sqrt{N}$ for thresholds corresponding to $0.5,0.1,0.01$, and 0.001 false alarm rates and 1000 independent samples averaged. Perfect $\left(N_{n} \gg N\right)$ noise subtraction is assumed in the power estimates and $\rho_{m}=1$.

where $\mathrm{SNR}_{c}=\operatorname{SNR} \rho_{m} /\left[\operatorname{SNR}\left(1-\rho_{m}\right)+1\right]$ treats the noncorrelated fraction of the signal as noise; for example, $\mathrm{SNR}_{c}$ for $\rho_{m}=0.8$ reaches a maximum value of 4 in the absence of noise. The parameters of this approximate expression for $B_{f}$ [Eq. (39)] were optimized to minimize the maximum deviation from simulated $B_{f}$ over $\mathrm{SNR}_{c} \sqrt{N}=-25$ to $+25 \mathrm{~dB}, N>10$, and $\rho_{m}>0.5$. Figure 6 shows that the CP bias can be approximated by the signal-free Rayleigh pdf-based noise bias [Eq. (20)] at low $\mathrm{SNR}_{c}$ and for $N>10$. At high $\mathrm{SNR}_{c}$, there is still a residual bias for small $N$.

The CP estimate mean, $\left\langle\hat{P}_{\mathrm{cp}}\right\rangle=\mu_{\mathrm{cp}}$, can be expressed using $B_{f}$ by rearranging Eq. (38) as

$$
\left\langle\hat{P}_{\mathrm{cp}}\right\rangle=\mu_{\mathrm{cp}}=\rho_{m} P_{s}+\frac{\sqrt{\pi}}{2 \sqrt{N}} B_{f}\left[\left(1-\rho_{m}\right) P_{s}+P_{n}\right] .
$$

The CP mean calculated using the $B_{f}$ estimate of Eq. (39) is within $3 \%$ of simulations for $N \geq 10$, evaluated for $\rho_{m}=1$ and 0.5. Using the combination of the Beckmann pdf of Eq. (33) (using five terms) when $\alpha / \sqrt{s_{1}+s_{2}}$ ) $\leq 5$, and the Gaussian pdf when $\left(\alpha / \sqrt{s_{1}+s_{2}}\right)>5$ is about $1 \%$ of the simulations for $N>50$, evaluated for $\rho_{m}=1$ and 0.5 .

The CP estimated standard deviation $\left(\sigma_{\mathrm{cp}}\right)$ can be directly calculated by combining Eqs. (32) and (40). The $\mathrm{CP}$ standard deviation calculated using the estimated $B_{f}$ [Eq. (39)] for $N>10$ is within $7 \%$ and using the Beckmann/Gaussian pdf-based estimate mean for $N>50$ is within $2 \%$ of the simulated $\sigma_{\mathrm{cp}}$ for $\rho_{m}>0.5$.

\section{c. PD comparison of CP and NSP techniques}

The $\mathrm{CP}$ probability of detection $\left(\mathrm{PD}_{\mathrm{cp}}\right)$ for large $N$ $(N=1000)$ and $\rho_{m}=1$ was found from the simulations, and the results are plotted in Fig. 7, along with the PD of NSP with perfect noise subtraction, for various false alarm rates (threshold levels). It is important to note that at a low threshold level (PFA $>10 \%$ ), the probability of detection with the NSP technique can be better than CP, but at higher threshold levels (lower PFA), CP is more likely to detect a weak signal. This was confirmed with measured data presented later, but the sensitivity difference between the CP and NSP techniques is not of practical significance. The more important benefit of the CP technique is its inherent noise-canceling property.

\section{Coherent power bias at low SNR}

The signal-free CP bias is $(\sqrt{\pi} / 2)\left(P_{n} / \sqrt{N}\right)$ for large $N$ [Eq. (20)]. With increasing SNR and $N$, this bias diminishes and the $\mathrm{CP}$ estimate converges to the pulsepair signal component covariance magnitude [Eq. (40)]. The noise bias with increasing SNR was evaluated using the simulations assuming unity pulse-pair signal correlation magnitude $\left(\rho_{m}=1\right)$ and was expressed as the ratio of CP to signal power as a function of $10 \log (\operatorname{SNR} \sqrt{N})$ in Fig. 8.

It usually makes sense to remove bias from data, but in this case there is rarely a practical benefit. CP bias is significant only for SNRs below low threshold levels (corresponding to about $10 \%$ or greater false alarm rates), but more importantly $\mathrm{CP}$ estimates at these low $\operatorname{SNR} \sqrt{N}$ have a high standard deviation relative to the mean $\left[\lim _{\mathrm{SNR} \sqrt{N} \rightarrow 0} \frac{\sigma_{\mathrm{cp}}}{\mu_{\mathrm{cp}}}=\sqrt{\frac{4}{\pi}-1} \approx 0.5\right.$, from Eqs. (20), (21)], 


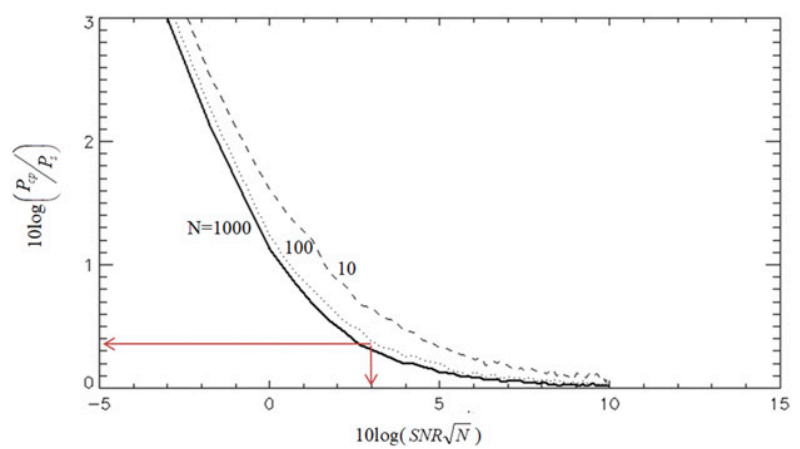

FIG. 8. Simulated ratio of $\mathrm{CP}$ to signal power as a function of $\operatorname{SNR} \sqrt{N}$ for $\rho_{m}=1$ and $N=1000$ (solid line), 100 (dots), and 10 (dashes). Shown is a $0.35 \mathrm{~dB}$ bias at $3 \mathrm{~dB}$ SNR $\sqrt{N}$ for 100 samples averaged (arrows).

so the practical value of these data samples is limited with or without bias correction. Therefore, the following bias correction procedure is given for completeness and for further information on the underlying $\mathrm{CP}$ estimate uncertainty (residual bias). This correction procedure does not suggest that it needs to be implemented or that it will improve the quality of $\mathrm{CP}$ estimates.

The bias correction procedure is similar to the NSP technique, in that it requires the estimation of the signalfree CP mean noise level. This CP noise floor can be directly measured, similar to the noise component of NSP, in a signal-free range gate(s). Range gates beyond the farthest scatterers can be used or the receiver measurement frequency band can be shifted away from the transmitted signal frequency to ensure signal-free samples. With zenith- or nadir-pointing radars like KPR, the PRI can be increased to sample range gates below the ground return and above the highest scatterers above the aircraft. However, unlike the noise component in signalplus-noise power measurements, $\mathrm{CP}$ bias is a function of SNR, so this correction is slightly more complicated. First, the ratio of biased $\mathrm{CP}$ to signal-free $\mathrm{CP}$ bias is calculated. The ratio of these two measurements is referred to as coherent power-to-signal-free $\mathrm{CP}$ bias ratio (CBR).

$$
\mathrm{CBR}=\frac{\left\langle\widehat{P}_{\mathrm{cp}}\right\rangle}{\frac{\sqrt{\pi} P_{n}}{2 \sqrt{N}}}
$$

Next, the SBR is defined as the coherent signal component-to-signal-free $\mathrm{CP}$ bias ratio (SBR), and a function will be found to relate CBR to SBR:

$$
\mathrm{SBR}=\frac{\rho_{m} P_{s}}{\frac{\sqrt{\pi} P_{n}}{2 \sqrt{N}}}=f(\mathrm{CBR})
$$

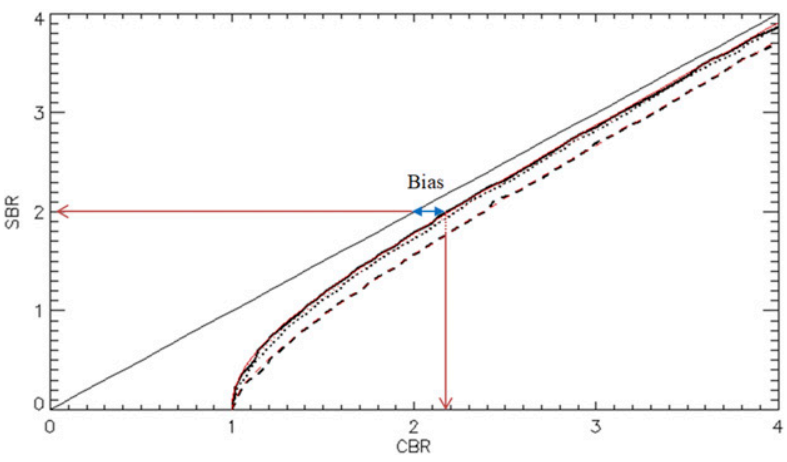

FIG. 9. Simulated CBR vs SBR (black lines), for SBR ranging from 0 to 4, along with the approximate formula relating CBR to SBR [Eq. (44)] (red lines) for $N=1000$ (solid), 100 (dots), and 10 (dashes). Unit slope line is drawn as the unbiased reference. Horizontal distance between this reference line and CBR is the bias.

When SBR is multiplied by the already known signalfree $\mathrm{CP}$ bias, the unbiased $\mathrm{CP}$ signal component is obtained:

$$
\rho_{m} P_{s}=\operatorname{SBR} \frac{\sqrt{\pi} P_{n}}{2 \sqrt{N}}
$$

Estimating CP noise bias is complicated because of the shifting CP pdf from Rayleigh toward chi-squared (Gaussian for large $N$ ) as SNR increases. We therefore again used the simulations to characterize the relationship between CBR and SBR as a function of SNR $\sqrt{N}$. Examining the simulated CBR and SBR relationship, we found that the following equation is a good approximation for $N>50$ :

$\mathrm{SBR}=f(\mathrm{CBR})=\left(\mathrm{CBR}^{2.25-\frac{2}{\sqrt{N}}-1}\right)^{\frac{1}{2.25-\frac{2}{\sqrt{N}}}}$

The comparison of simulated and the Eq. (44) model relationship between SBR and CBR is shown in Fig. 9. Note that CP bias is only about $10 \%(0.4 \mathrm{~dB})$ when SBR is $3 \mathrm{~dB}(\mathrm{SNR} \sqrt{N}=\sim 2.5 \mathrm{~dB})$, and it further decreases with increasing $\operatorname{SNR} \sqrt{N}$.

\section{Coherent power bias as a result of spectrum width}

Equations (25) and (40) show that the CP estimate is approximately equal to $\rho_{m} P_{s}$ at high SNR and when a large number of samples are averaged. ${ }^{2}$ The $\mathrm{CP}$ bias

\footnotetext{
${ }^{2}$ From Eq. (40), if $\frac{1}{\sqrt{N}}\left[\left(1-\rho_{m}\right)+\frac{1}{\mathrm{SNR}}\right]<0.02$ and $\rho_{m}>0.5$, then $10 \log \left(\frac{\widehat{P}_{\mathrm{cp}}}{\rho_{m} P_{s}}\right)<\sim 0.1 \mathrm{~dB}$.
} 


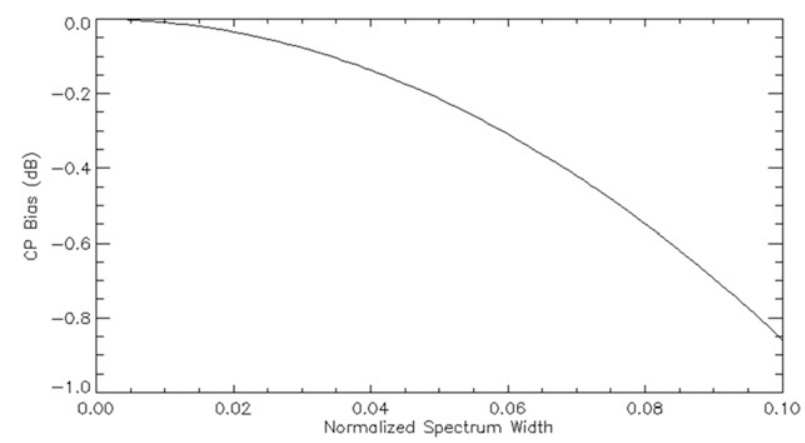

FIG. 10. High SNR and unit $m$ CP bias (dB), as a function of normalized spectrum width. Equation $10 \log \left(\rho_{1}\right)=-8.68\left(\pi \sigma_{v n}\right)^{2}$ is plotted for $\sigma_{v n}=0-0.1$.

associated with $\rho_{m}$ can be estimated from multiple lag pulse-pair measurements, without the need to estimate the noise component of the signal-plus-noise measurements (Lei et al. 2012; Cao et al. 2012). The normalized signal correlation of weather targets can be approximated as (Doviak and Zrnić 1993, p. 125)

$$
\begin{aligned}
\rho_{m} & \approx \exp \left[-8\left(\pi \sigma_{v} m T_{s / \lambda}\right)^{2}\right] \\
& =\exp \left[-2\left(\pi \sigma_{v n} m\right)^{2}\right] .
\end{aligned}
$$

The lag- $m T_{s}$ coherent power to the signal power ratio, for large $N$ and SNR, is the corresponding correlation coefficient, $\rho_{m}$ :

$$
\begin{aligned}
\rho_{m} & \approx \frac{P_{\mathrm{cp}}\left(m T_{s}\right)}{P_{s}}=\frac{\left\langle[I(k)+j Q(k)]^{*}[I(k+m)+j Q(k+m)]\right\rangle \mid}{\left\langle|I+j Q|^{2}\right\rangle} \\
& \approx \exp \left[-2\left(\pi \sigma_{v n} m\right)^{2}\right] .
\end{aligned}
$$

This high SNR CP bias estimate $(\mathrm{dB})$ as a function of normalized spectrum width for $m=1$ is illustrated in Fig. 10.

To estimate $\rho_{m}$ from multiple lag pulse-pair measurements, only $\sigma_{v n}$ needs to be approximated. If we measure the lag- $m_{1} T_{s}$ and lag- $m_{2} T_{s}$ coherent power $\left(m_{1} \neq m_{2}, \rho_{m_{1}}>0\right.$ and $\left.\rho_{m_{2}}>0\right)$, then

$$
\begin{gathered}
\frac{P_{\mathrm{cp}}\left(m_{1} T_{s}\right)}{P_{\mathrm{cp}}\left(m_{2} T_{s}\right)} \approx \frac{\rho_{m_{1}}}{\rho_{m_{2}}} \approx \exp \left[2 \pi^{2} \sigma_{v n}^{2}\left(m_{2}^{2}-m_{1}^{2}\right)\right], \quad \text { and } \\
\sigma_{v n} \approx \frac{1}{\pi} \sqrt{\frac{\ln \left[\frac{P_{\mathrm{cp}}\left(m_{1} T_{s}\right)}{P_{\mathrm{cp}}\left(m_{2} T_{s}\right)}\right]}{2\left(m_{2}^{2}-m_{1}^{2}\right)} .}
\end{gathered}
$$

With $\sigma_{v n}$, the coherent power negative bias $(\mathrm{dB})$ as a result of spectrum width can be estimated using Eq. (46):

$$
\begin{gathered}
10 \log _{10}\left[\frac{P_{s}}{P_{\mathrm{cp}}\left(m T_{s}\right)}\right] \\
\operatorname{large} N \text { and SNR. }
\end{gathered}
$$

This method, using lag-50- and lag-150- $\mu$ s pulse spacing, was tested on cloud and precipitation data collected with the UWyo KPR radar, and the results agree within $0.1 \mathrm{~dB}$ with the bias estimated by directly computing the ratio of the measured noise-subtracted power and $\mathrm{CP}$ as described in section 6 .

\section{Experimental results}

The radar reflectivity factor was estimated from data collected using the conventional NSP and CP techniques with KPR, on board the University of Wyoming King Air 200T research aircraft (Fig. 11). KPR is a compact, dualbeam, solid-state Doppler radar that operates from a standard Particle Measurement Systems (PMS) canister. A $10-\mathrm{W}$ peak-power solid-state amplifier is used to transmit pulses of a chirped (frequency modulated) waveform, immediately followed by an offset frequency short pulse for close-range measurements. The received signals from both pulse segments are simultaneously

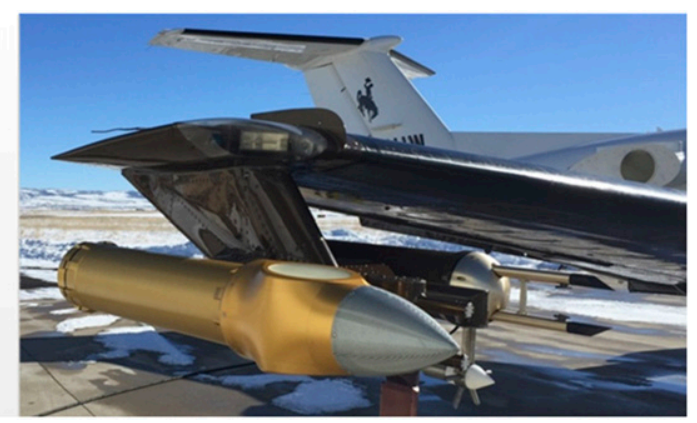

FIG. 11. UWyo KPR is a compact, dual-beam Doppler radar that operates from a standard PMS probe canister. 


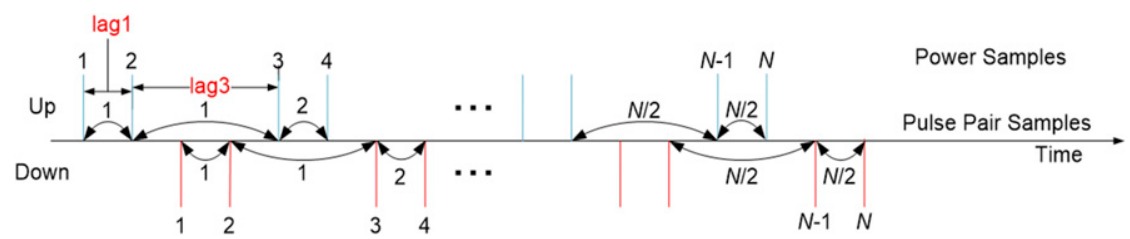

FIG. 12. KPR interleaved up-/down-looking antenna pulsing sequence. For every $N$ power samples collected per averaging interval, $N / 2$ pulse pairs are available for processing at lag- 1 and lag-3 spacing. Lag-1 and lag-3 pulse pairs are averaged to obtain the same number of pulsepair noise samples $(N)$ as NSP, but the lag-3 pulse-pair phase is first divided by 3 to match the lag-1 phase.

measured with a wide bandwidth front-end and dualchannel back-end receiver sections. KPR can operate in a variety of modes, including pulse-pair and full spectrum processing. When using a single up- or down-looking antenna, constant PRI is employed. When simultaneous upward and downward pulse-pair measurements are required, the system can be configured to alternately transmit pairs of pulses to the up- and down-looking antennas, as illustrated in Fig. 12. The real-time computed radar data products include radar reflectivity factor and Doppler velocity moments from pulse pairs. A summary of the KPR system's parameters is listed in Table 1.

The dataset presented in Figs. 13-19 was collected on 4 September 2016 in southeastern Wyoming, about $50 \mathrm{~km}$ east of Rawlins. The aircraft was flying through convective cells approximately $4 \mathrm{~km}$ above ground and $\sim 2.2 \mathrm{~km}$ above the melting layer in $-11^{\circ} \mathrm{C}$ flight-level temperature. The radar PRF was a constant $20 \mathrm{kHz}$, transmitting alternating pairs of pulses to the up- and down-looking antennas as shown in Fig. 12. The resulting lag-1 and lag-3 pulse-pair spacings were 50 and $150 \mu$ s, respectively. Each pulse contained a Tukey tapered $2.5-\mu$ s-long $5-\mathrm{MHz}$ bandwidth chirped pulse, immediately followed by a similarly tapered $0.25-\mu$ s short RF pulse at an offset frequency. The resulting range resolution of the compressed chirp and short radio frequency (RF) pulses was approximately $35 \mathrm{~m}$. The up- and down-pointed beams and short and compressed chirped pulse datasets were combined such that the short-pulse data were used to about $800 \mathrm{~m}$ from the aircraft and the chirped data, with better sensitivity, beyond. Each noise-subtracted power and coherent power reflectivity point was calculated by averaging 2000 samples (0.2-s integration time; five profiles per second per beam). The signal components of the samples were partially correlated as a result of the high $\mathrm{PRF}$, so the equivalent number of independent high-SNR signal samples was only about $200 .{ }^{3}$ To obtain the same

\footnotetext{
${ }^{3}$ The measured normalized spectrum width of this dataset is approximately $2.5 \mathrm{~m} \mathrm{~s}^{-1} / 84 \mathrm{~m} \mathrm{~s}^{-1}=0.03$, and from Doviak and Zrnić (1993, p. 128) $N_{I} \approx 2 N \sigma_{v n} \sqrt{\pi}=211$, for large $N$ and $\sigma_{v n} \ll 1$.
}

(2000) number of pulse-pair samples per CP reflectivity estimate, the lag-3 pulse-pair phasor phase was divided by 3 and then the lag- 1 and lag-3 phasors were averaged.

The average of 2000 signal-plus-noise power samples yields a zero SNR measurement standard deviation of about $16.5 \mathrm{~dB}$ below the mean noise power. Thus, even with a relatively high (for a research weather radar) threshold factor of 2.5 (4. $\mathrm{dB}$ and $\sim 1 \%$ PFA), the detection threshold is about $12.5 \mathrm{~dB}$ below the mean noise floor. To maintain close to this measurement precision and sensitivity using the NSP technique, the noise component was estimated by averaging the last 20 (signal free) range gates of each averaged ray, totaling 40000 samples, to estimate the noise component of each signal-plus-noise measurement. The resulting noise component estimate standard deviation is $P_{n} / \sqrt{40000}$, or the uncertainty relative to the noise power:

$$
10 \log \left(\frac{P_{n} \pm \frac{P_{n}}{\sqrt{40000}}}{P_{n}}\right) \approx \pm 0.02 \mathrm{~dB} .
$$

The accuracy of the noise component estimate can be worse however because of the bias caused by receiver gain variation in range or radar echo. Noise estimate uncertainties as high as $2 \mathrm{~dB}$ have been cited by Keranen and Chandrasekar (2014) with operational weather radars. This is likely excessive with most research radars, but nevertheless estimating the noise component to about $0.02-\mathrm{dB}$ uncertainty is not a trivial requirement. This makes the CP technique particularly attractive with KPR, and without the need for signal-free range gates for noise subtraction, $\mathrm{PRF}$ can be increased to maximize sensitivity. The staggered PRI mode shown in Fig. 12 has the added benefit that second-trip echo is uncorrelated in the CP estimate and therefore the associated bias is reduced with averaging according to Eq. (40).

The CP reflectivity image, without thresholding or bias correction, is shown in Fig. 13 and the corresponding SNR in Fig. 14. In Fig. 13 the zero SNR bias is clearly visible outside the cloud and precipitation regions. The mean and standard deviation of the $\mathrm{CP}$ signal-free noise 
TABLE 1. KPR key system parameters.

Frequency

Transmitter power

Transmitter loss

Transmit pulse width

Transmit waveform

Transmit polarization

PRF

Antennas

Antenna bandwidth

Radome

Antenna

Antenna gain

First sidelobe level

Receiver type

Receiver noise figure

Range gate spacing

Radar intermediate frequency

Digital receiver

Dynamic range

Processor
$35.64 \mathrm{GHz}+-30 \mathrm{MHz}$

10 -W peak, $\sim 5 \%$ duty (power supply limited)

$\sim 1 \mathrm{~dB}$

$0.1-20 \mu \mathrm{s}$

Interleaved chirped long pulse/short pulse

Linear

Up to $20 \mathrm{kHz}$

Up- and down-pointed, linear-polarized flat-plate arrays $35.5-35.9 \mathrm{GHz}$

Rexolite (0.1-dB loss one way)

14-cm diameter, $4.2^{\circ}$ half-power beamwidth

$32.5 \mathrm{~dB}$

$-23 \mathrm{~dB}$

Single wideband RF; dual IF

$\sim 4 \mathrm{~dB}$

$7.5-75 \mathrm{~m}$

90/150 MHz

Dual-channel, 16-bit analog-to-digital converter (ADC) $90 \mathrm{~dB}$ at $1-\mathrm{MHz}$ bandwidth

Industrial PC, dual Hex Core 2.3-GHz Xeon floor in Eqs. (20) and (21) for large $N$ are confirmed in the upper-right signal-free rectangular region. The mean measured CP bias to $P_{n} / \sqrt{N}$ ratio is 0.885 (0.002 standard deviation), compared to the theoretical $(\sqrt{\pi} / 2) \approx 0.886$, and the measured standard deviation to $P_{n} / \sqrt{N}$ ratio is 0.46 (approximately $\pm 0.007,90 \%$ confidence interval), compared to $\sqrt{1-(\pi / 4)} \approx 0.463$.

The $50 \%$ false alarm rate $\mathrm{CP}$ with bias correction and NSP images are shown in Figs. 15 and 16, respectively. The correct thresholding (50\% PFA) in both images is confirmed by the PFA in the signal-free regions in the upper-right corner (0.499 CP and 0.501 NSP PFA; $\sim 0.002$ standard deviation). The slightly better sensitivity of the NSP technique at low threshold levels (high PFA), indicated in Fig. 7, is confirmed by a slightly higher power PD $(0.87 ; \sim 0.017$ standard deviation) than the detection rate of $\mathrm{CP}(0.82 ; \sim 0.017$ standard deviation $)$ in the upper-left corner test region. When the threshold level is increased, so the PFA is close to $0.1 \%$, in Figs. 17 and 18, the $\mathrm{CP}$ detection rate $(0.91 ; \sim 0.011$ standard deviation $)$ is slightly higher than the power PD $(0.85 ; \sim 0.011$ standard deviation), in a test region at the 4-km range above the aircraft and in the left part of the images, as predicted by the analytical and simulation results plotted in Fig. 7.

The last image presented, in Fig. 19, compares the multilag [using Eq. (49)] to the direct NSP-to-CP ratio method of estimating $\mathrm{CP}$ bias due to spectrum width. In this dataset, the multilag method agrees with the direct NSP-to-CP ratio to within $\sim 0.1 \mathrm{~dB}$.

\section{Conclusions}

The CP and NSP techniques were shown to have similar sensitivity and accuracy to measure weather radar signals when properly implemented. The fact that the CP technique does not require an independent noise estimate can be very useful, particularly with radars that average a large number of samples and consequently extend the measurement dynamic range well below unity SNR, where the required noise component measurement accuracy is increasingly difficult to achieve. On the other hand, the $\mathrm{CP}$ technique requires the pulse-pair spacing to

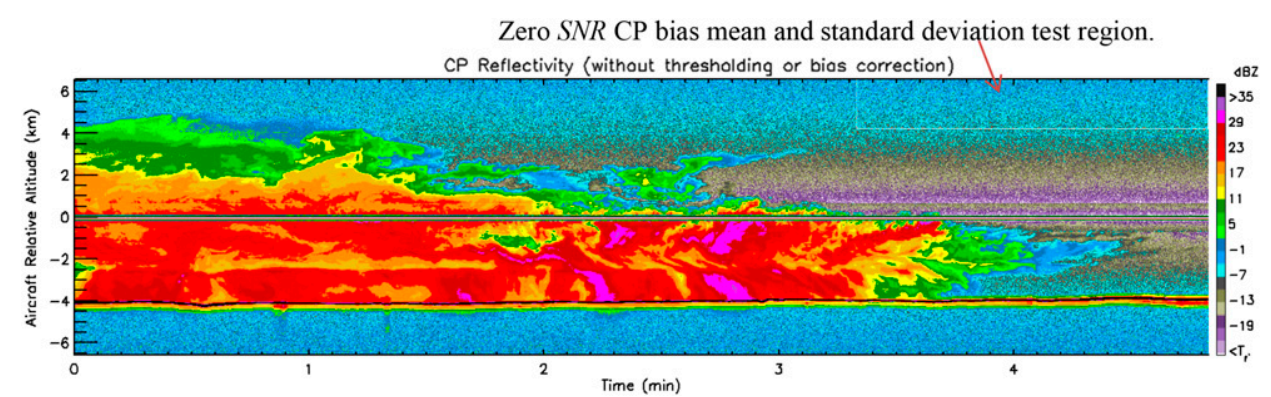

FIG. 13. CP reflectivity image, without thresholding or bias correction. CP bias mean and standard deviation, in the upper-right corner test region, agree with Eqs. (17) and (18). 


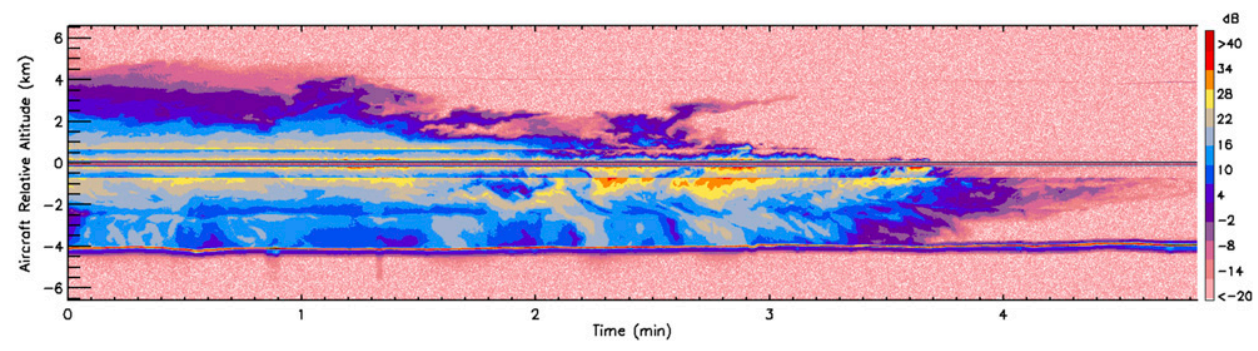

FIG. 14. CP SNR (dB).

be much less than the inverse of the signal bandwidth to ensure close to unity signal component correlation. In addition, some thresholding should be applied to mask low SNR CP bias. Properly collected CP data are actually slightly more sensitive to weak signals than the power technique when the threshold level is set high (low false alarm rate), but the more important benefit of the $\mathrm{CP}$ technique is its inherent noise-canceling property.

The coherent power estimate probability density function for a high number of samples ( $N$ and $N_{I}$ ) has been identified to be the Beckmann distribution, which converges to the Rayleigh pdf in the special case when the pulse-to-pulse signal correlation or SNR is zero, and to Gaussian pdf at high SNR and $\rho_{m} \approx 1$. A simple expression for CP standard deviation has been provided [Eq. (32)] and an approximate expression for CP mean has been found [Eq. (40)] that agrees within $3 \%$ of simulated $\mathrm{CP}$ estimate mean and, as part of Eq. (32), agrees within 7\% for the standard deviation.

Data collected with the University of Wyoming airborne Ka-band precipitation radar (KPR) confirm the analytical and simulation results of zero SNR CP residual noise and the comparison between CP and NSP techniques. The CP residual noise floor mean and the standard deviation of Eqs. (20) and (21) for a large number of samples are in close agreement with calculations using Eq. (19) (Lee et al. 1994), simulations. and measurements, while it was found that the expressions of Eq. (A10) in Keranen and Chandrasekar (2014) are slightly biased estimates. Equation (23), for computing the required threshold level to achieve a specific false alarm rate, has also been confirmed. The CP signal power measurements agree to within $0.1 \mathrm{~dB}$ with the NSP measurements after correcting the effects of spectrum width using the multilag technique outlined in this paper. The combination of analytical, simulation, and experimental results confirm that $\mathrm{CP}$ can be a more accurate alternative to the NSP technique at low SNR and when the noise component cannot be estimated at an accuracy that is considerably better than that of the signal-plus-noise measurements.

Acknowledgments. The authors thank Dr. James Mead of ProSensing, Inc., for the helpful discussions and suggestions related to the analyses presented herein, and the AMS reviewers for their thorough review and numerous constructive suggestions. The development of KPR and the data collection effort were funded by NASA EPSCoR Grant NNX13AN09A to the University of Wyoming.

\section{APPENDIX}

\section{Statistics of the Real and Imaginary Components of Pulse-Pair Average}

Coherent power is estimated by averaging lag- $m T_{s}$ pulse-pairs. In this appendix the mean and variance of the real and imaginary components $\left(Q_{\mathrm{cp}}\right.$ and $\left.I_{\mathrm{cp}}\right)$ of this average are calculated, assuming $\varphi_{D}=0$ :

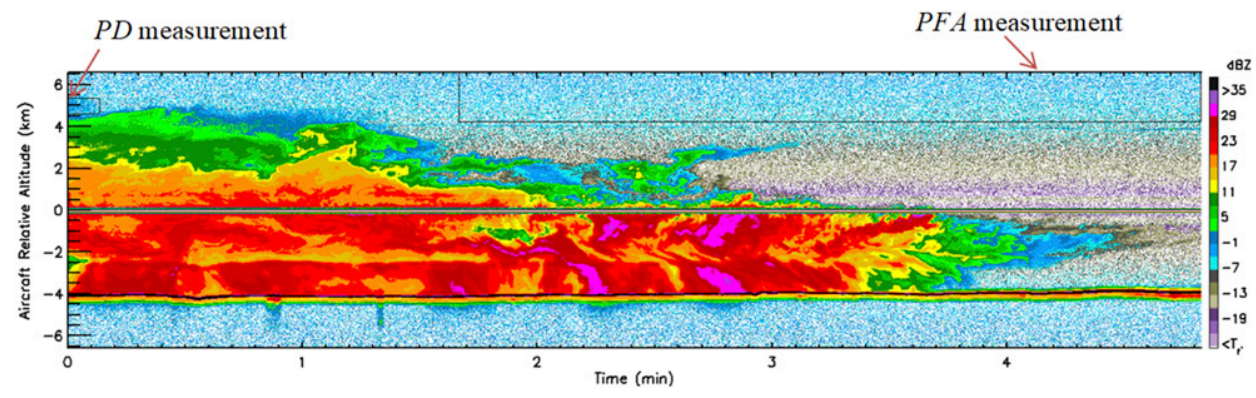

FIG. 15. Noise-bias-corrected CP reflectivity image, with thresholding that corresponds to $50 \%$ false alarm rate using Eq. (20). False alarm rate of the signal-free region inside the black rectangle in the upperright corner is 0.499 , confirming the correct thresholding. PD in the small upper-left rectangle is 0.821 , compared to 0.870 in the same region in the NSP image of Fig. 16. 


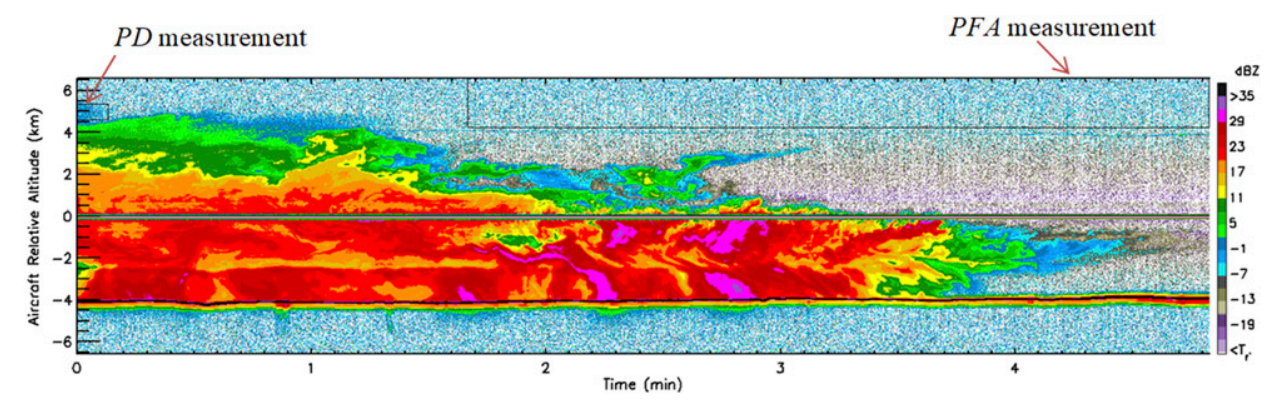

FIG. 16. NSP reflectivity, without thresholding $\left(T_{f}=0 ; 50 \% \mathrm{PFA}_{P}\right)$. False alarm rate of a signal-free region inside the black rectangle in the upper-right corner is 0.498 (50\% expected). PD in the small upperleft rectangle is 0.870 compared to 0.821 in the same region in the CP reflectivity image of Fig. 15 , confirming that the power technique is slightly more sensitive to weak signals than CP when the PFA is high (at low threshold) in Fig. 7.

$$
\hat{P}_{\mathrm{cp}}=\left|\frac{1}{N} \sum_{k=1}^{N} V^{*}(k) V(k+m)\right|=\left|I_{\mathrm{cp}}+j Q_{\mathrm{cp}}\right|,
$$

where

$$
\begin{aligned}
V(k)= & V_{s}(k)+V_{n}(k)=I_{s}(k)+j Q_{s}(k) \\
& +I_{n}(k)+j Q_{n}(k) \text { and } m \ll N .
\end{aligned}
$$

The signal $\left(I_{s}, Q_{s}\right)$ and noise $\left(I_{n}, Q_{n}\right)$ terms are zeromean Gaussian random variables (RV) with variance $\sigma_{s}^{2}=P_{s} / 2$ and $\sigma_{n}^{2}=P_{n} / 2$, respectively. Furthermore, $\left\langle V_{s}^{*}(k) V_{s}(k+m)\right\rangle=\rho_{m} P_{s}, \quad\left\langle V_{s} V_{n}\right\rangle=0, \quad$ and $\left\langle V_{n}^{*}(k) V_{n}(k+m)\right\rangle=0$. In terms of the signal and noise voltage samples, the coherent power estimate can be expanded as

$$
\hat{P}_{\mathrm{cp}}=|| \frac{1}{N} \sum_{k=1}^{N}\left[V_{s}^{*}(k) V_{s}(k+m)+V_{s}^{*}(k) V_{n}(k+m)+V_{n}^{*}(k) V_{s}(k+m)+V_{n}^{*}(k) V_{n}(k+m)\right] \mid,
$$

where

the signal-signal terms are $\frac{1}{N} \sum_{k=1}^{N} V_{s}^{*}(k) V_{s}(k+m)=\hat{I}_{\mathrm{ns}}+j \hat{Q}_{\mathrm{ns}}, \quad$ and

$\hat{I}_{\mathrm{ss}}+j \hat{Q}_{\mathrm{ss}}$,

the signal-noise terms are $\frac{1}{N} \sum_{k=1}^{N} V_{s}^{*}(k) V_{n}(k+m)=$

$\hat{I}_{\mathrm{sn}}+j \hat{Q}_{\mathrm{sn}}$,

the noise-noise terms are $\frac{1}{N} \sum_{k=1}^{N} V_{n}^{*}(k) V_{n}(k+m)=$ $\hat{I}_{\mathrm{nn}}+j \hat{Q}_{\mathrm{nn}}$

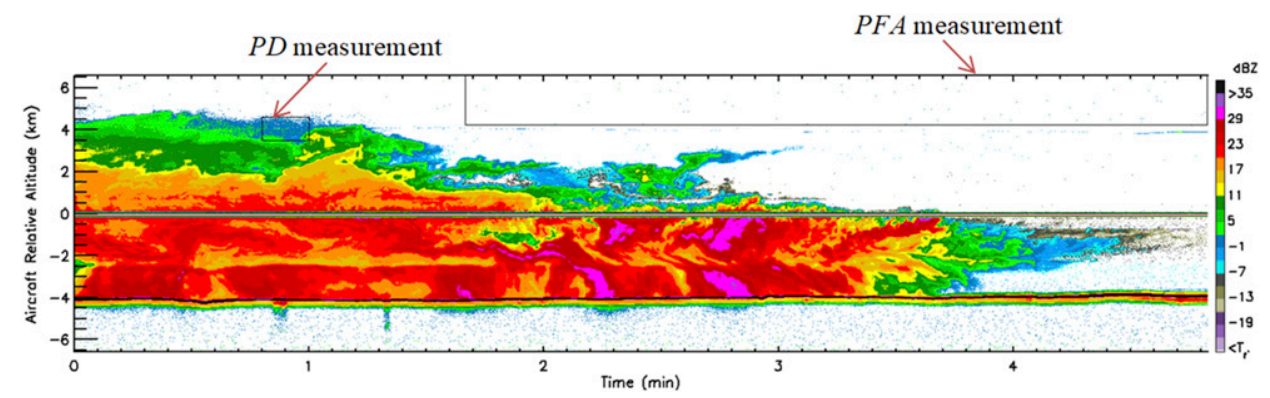

FIG. 17. CP reflectivity (noise bias corrected), with thresholding set to yield $0.1 \%$ false alarm rate using Eq. (20). False alarm rate of the signal-free region inside the rectangle in the upper-right corner was evaluated to be 0.0011 ( 0.0013 in the power image of Fig. 18). PD in the small upper-left rectangle is 0.906 compared to 0.850 in the same region using NSP in Fig. 18, confirming higher CP sensitivity to weak signals than the power technique at lower false alarm rates, as indicated in Fig. 7, in spite of the approximately 0.5-dB lower CP SNR as a result of nonzero spectrum width (Fig. 19). 


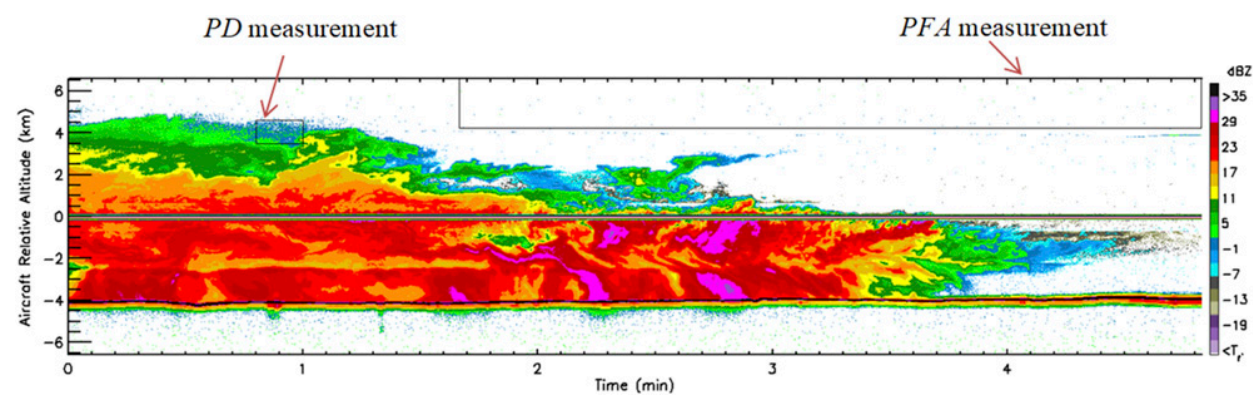

FIG. 18. NSP reflectivity, with thresholding set to yield a $0.1 \%$ false alarm rate using Eq. (14). False alarm rate of a signal-free region inside the black rectangle in the upper-right corner was evaluated to be 0.0013 ( 0.0011 in the CP image of Fig. 17). PD in the small upper-left rectangle is 0.850 compared to 0.906 in the same region in the CP image of Fig. 17.

\section{a. Noise-noise terms}

The noise-noise terms can be expanded as

$$
\begin{aligned}
\frac{1}{N} \sum_{k=1}^{N} V_{n}^{*}(k) V_{n}(k+m) \\
=\frac{1}{N} \sum_{k=1}^{N}\left[I_{n}(k) I_{n}(k+m)+Q_{n}(k) Q_{n}(k+m)\right. \\
\left.\quad+j I_{n}(k) Q_{n}(k+m)-j I_{n}(k+m) Q_{n}(k)\right] .
\end{aligned}
$$

Each product term has a variance of $\left(\sigma_{n}^{2}\right)^{2}=P_{n}^{2} / 4$, and since there are $2 N$ real and $2 N$ imaginary terms, $\hat{I}_{\mathrm{nn}}$ and $\hat{Q}_{\mathrm{nn}}$ are zero-mean $\mathrm{RVs}$ with variance $2 \sigma_{n}^{4} / N=P_{n}^{2} / 2 N$.

\section{b. Signal-noise and noise-signal terms}

Similarly, $\hat{I}_{\mathrm{sn}}, \hat{I}_{\mathrm{ns}}, \hat{Q}_{\mathrm{sn}}$, and $\hat{Q}_{\mathrm{ns}}$ are zero-mean RVs with variance $2 \sigma_{s}^{2} \sigma_{n}^{2}=P_{s} P_{n} / 2 N$.

\section{c. Signal-signal terms}

The signal portion of the voltage is separated into coherent and noncoherent components:

$$
\begin{gathered}
V_{s}=V_{\mathrm{sc}}+V_{\mathrm{si}}=\sqrt{\rho_{m}} I_{\mathrm{sc}}+j \sqrt{\rho_{m}} Q_{\mathrm{sc}}+\sqrt{1-\rho_{m}} I_{\mathrm{si}} \\
+j \sqrt{1-\rho_{m}} Q_{\mathrm{si}}, \quad \text { such that } \\
\left\langle V_{\mathrm{sc}}^{*}(k) V_{\mathrm{sc}}(k+m)\right\rangle=\rho_{m} P_{s} \text { and }\left\langle V_{\mathrm{si}}^{*}(k) V_{\mathrm{si}}(k+m)\right\rangle=0 .
\end{gathered}
$$

Again, we can define the real and imaginary components of the averages as

$$
\begin{aligned}
& \frac{1}{N} \sum_{k=1}^{N} V_{\mathrm{sc}}^{*}(k) V_{\mathrm{sc}}(k+m)=\hat{I}_{\mathrm{ssc}}+j \hat{Q}_{\mathrm{ssc}}, \\
& \frac{1}{N} \sum_{k=1}^{N} V_{\mathrm{si}}^{*}(k) V_{\mathrm{sc}}(k+m)=\hat{I}_{\mathrm{ssic}}+j \hat{Q}_{\mathrm{ssic}}, \\
& \frac{1}{N} \sum_{k=1}^{N} V_{\mathrm{sc}}^{*}(k) V_{\mathrm{si}}(k+m)=\hat{I}_{\mathrm{ssci}}+j \hat{Q}_{\mathrm{ssci}}, \quad \text { and } \\
& \frac{1}{N} \sum_{k=1}^{N} V_{\mathrm{si}}^{*}(k) V_{\mathrm{si}}(k+m)=\hat{I}_{\mathrm{ssi}}+j \hat{Q}_{\mathrm{ssi}} .
\end{aligned}
$$

The coherent part can be expressed as

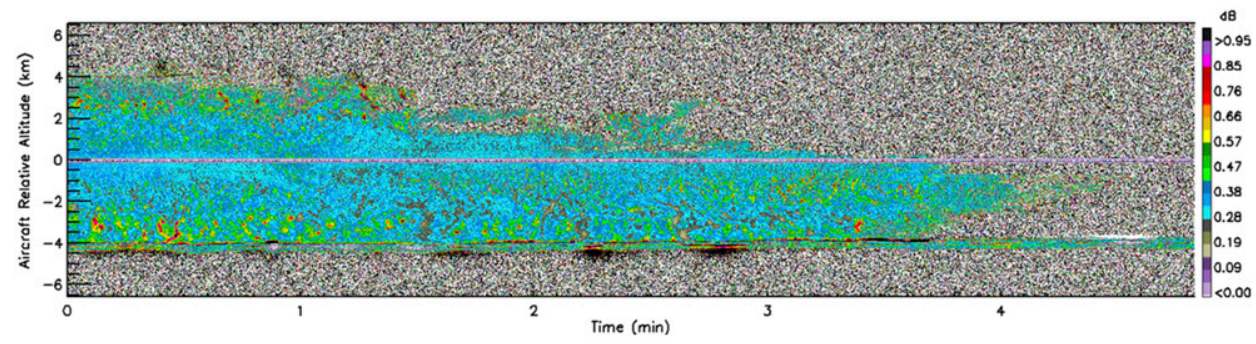

FIG. 19. Lag-1/lag-3 estimated CP negative bias and loss of SNR caused by aircraft motion and turbulence using Eq. (49). This agrees to less than $0.1 \mathrm{~dB}$ with the bias calculated directly by taking the ratio of NSP and CP. 


$$
\frac{1}{N} \sum_{k=1}^{N} V_{\mathrm{sc}}^{*}(k) V_{\mathrm{sc}}(k+m)=\frac{1}{N}\left[\sum_{k=1}^{N} \rho_{m} I_{\mathrm{sc}}^{2}(k)+\rho_{m} Q_{\mathrm{sc}}^{2}(k)+j \rho_{m} I_{\mathrm{ssc}}(k) Q_{\mathrm{ssc}}(k)-j \rho_{m} I_{\mathrm{ssc}}(k) Q_{\mathrm{ssc}}(k)\right],
$$

where the real part of each term is equivalent to the power sample with exponential distribution, and the average $\left(\hat{I}_{\mathrm{ssc}}\right)$ therefore has mean $\rho_{m} P_{s}$ and variance $\sigma_{\mathrm{ssc}}^{2}=\rho_{m}^{2} P_{s}^{2} / N_{I}$, where $N_{I}\left(N_{I} \gg \mathrm{m}\right)$ is the equivalent number of independent samples, while the imaginary parts cancel, so $\hat{Q}_{\mathrm{ssc}}=0$.

The cross terms are identical, so $\hat{I}_{\text {ssci }}, \hat{I}_{\text {ssic }}, \hat{Q}_{\text {ssci }}$, and $\hat{Q}_{\text {ssic }}$ are zero-mean $\mathrm{RVs}$ with variance $\rho_{m}\left(1-\rho_{m}\right) 2 \sigma_{s}^{4}=\rho_{m}\left(1-\rho_{m}\right)\left(P_{s}^{2} / 2 N\right)$.

The noncoherent portion of the signal is just like noise, so $\hat{I}_{\text {ssi }}$ and $\hat{Q}_{\text {ssi }}$ are zero-mean $\mathrm{RVs}$ with variance $\left(1-\rho_{m}\right)^{2} 2 \sigma_{s}^{4}=\left(1-\rho_{m}\right)^{2}\left(P_{s}^{2} / 2 N\right)$.

There is only one term with nonzero mean: $\left\langle\hat{I}_{\mathrm{ss}}\right\rangle=\rho_{m} P_{s}$. The variance of the signal-signal component is found by summing the variances of the terms:

$$
\begin{aligned}
\operatorname{var}\left(\hat{I}_{\mathrm{ss}}\right)= & \operatorname{var}\left(\hat{I}_{\mathrm{ssc}}\right)+\operatorname{var}\left(\hat{I}_{\mathrm{ssci}}\right) \\
& +\operatorname{var}\left(\hat{I}_{\mathrm{ssic}}\right)+\operatorname{var}\left(\hat{I}_{\mathrm{ssi}}\right) \\
\operatorname{var}\left(\hat{I}_{\mathrm{ss}}\right)= & \frac{\rho_{m}^{2} P_{s}^{2}}{N_{I}}+2 \rho_{m}\left(1-\rho_{m}\right) \frac{P_{s}^{2}}{2 N}+\left(1-\rho_{m}\right)^{2} \frac{P_{s}^{2}}{2 N} \\
= & \frac{\rho_{m}^{2} P_{s}^{2}}{N_{I}}+\left(1-\rho_{m}^{2}\right) \frac{P_{s}^{2}}{2 N}, \quad \text { and } \\
\operatorname{var}\left(\hat{Q}_{\mathrm{ss}}\right)= & \left(1-\rho_{m}^{2}\right) \frac{P_{s}^{2}}{2 N} .
\end{aligned}
$$

\section{d. Mean and variance of the pulse-pair sum terms}

There is only one non-zero-mean term: $\left\langle I_{\mathrm{cp}}\right\rangle=\rho_{m} P_{s}$, so $\left\langle Q_{\mathrm{cp}}\right\rangle=0$ and the variances can be summed as $\operatorname{var}\left(I_{\mathrm{cp}}\right)=\operatorname{var}\left(\hat{I}_{\mathrm{ss}}\right)+\operatorname{var}\left(\hat{I}_{\mathrm{sn}}\right)+\operatorname{var}\left(\hat{I}_{\mathrm{ns}}\right)+\operatorname{var}\left(\hat{I}_{\mathrm{nn}}\right)$, so

$\operatorname{var}\left(I_{\mathrm{cp}}\right)=\frac{\rho_{m}^{2} P_{s}^{2}}{N_{I}}+\frac{\left(1-\rho_{m}^{2}\right) P_{s}^{2}+2 P_{s} P_{n}+P_{n}^{2}}{2 N}$

and similarly

$$
\begin{aligned}
\operatorname{var}\left(Q_{\mathrm{cp}}\right)= & \operatorname{var}\left(\hat{Q}_{\mathrm{ss}}\right)+\operatorname{var}\left(\hat{Q}_{\mathrm{sn}}\right)+\operatorname{var}\left(\hat{Q}_{\mathrm{ns}}\right) \\
& +\operatorname{var}\left(\hat{Q}_{\mathrm{nn}}\right), \text { and } \\
\operatorname{var}\left(Q_{\mathrm{cp}}\right)= & \frac{\left(1-\rho_{m}^{2}\right) P_{s}^{2}+2 P_{s} P_{n}+P_{n}^{2}}{2 N}
\end{aligned}
$$

\section{REFERENCES}

Abramowitz, M., and I. A. Stegun, Eds., 1964: Handbook of Mathematical Functions: With Formulas, Graphs, and Mathematical Tables. Applied Mathematics Series, Vol. 55, National Bureau of Standards, 1046 pp.
Beckmann, P., 1962: Statistical distribution of the amplitude and phase of a multiply scattered field. J. Res. Natl. Bur. Stand., 66D, 231-240.

Bell, M. K., W. Lee, C. A. Wolff, and H. Cai, 2013: A solo-based automated quality control algorithm for airborne tail Doppler radar data. J. Appl. Meteor. Climatol., 52, 2509-2528, https:// doi.org/10.1175/JAMC-D-12-0283.1.

Cao, Q., G. Zhang, R. D. Palmer, M. Knight, R. May, and R. J. Stafford, 2012: Spectrum-Time Estimation and Processing (STEP) for improving weather radar data quality. IEEE Trans. Geosci. Remote Sens., 50, 4670-4683, https://doi.org/ 10.1109/TGRS.2012.2190608.

Doviak, R. J., and D. S. Zrnić, 1993: Doppler Radar and Weather Observations. 2nd ed. Academic Press, 562 pp.

Fortune, S., 2005: Phase error estimation for synthetic aperture imagery. Ph.D. thesis, University of Canterbury, 173 pp.

Goodman, J. W., 1975: Statistical properties of laser speckle patterns. Laser Speckle and Related Phenomena, J. C. Dainty, Ed., SpringerVerlag, 9-75.

Hildebrand, P. H., and R. S. Sekhon, 1974: Objective determination of the noise level in Doppler spectra. J. Appl. Meteor., 13, 808-811, https://doi.org/10.1175/1520-0450(1974) $013<0808$ :ODOTNL $>2.0$. CO;2.

Ivić, I. R., 2009a: Detection Thresholds for Spectral Moments and Polarimetric Variables. VDM Publishing, $212 \mathrm{pp}$.

_ D. S. Zrnić and T.-Y. Yu, 2009b: Use of coherency to improve signal detection in dual-polarization weather radars. J. Atmos. Oceanic Technol., 26, 2474-2487, https://doi.org/10.1175/2009JTECHA1154.1.

_ C. Curtis, and S. M. Torres, 2013: Radial-based noise power estimation for weather radars. J. Atmos. Oceanic Technol., 30, 2737-2753, https://doi.org/10.1175/JTECH-D-13-00008.1.

_, R. Keranen, and D. S. Zrnić, 2014a: Assessment of censoring using coherency-based detectors on dual-polarized weather radar. J. Atmos. Oceanic Technol., 31, 1694-1703, https://doi. org/10.1175/JTECH-D-13-00074.1.

, J. C. Krause, O. E. Boydstun, A. E. Daniel, A. D. Free, and W. D. Zittel, 2014b: Effects of radial-based noise power estimation on spectral moments. J. Atmos. Oceanic Technol., 31, 2671-2691, https://doi.org/10.1175/JTECH-D-14-00048.1.

Jentschura, U. D., and E. Lötstedt, 2011: Numerical calculation of Bessel, Hankel and Airy functions. Comput. Phys. Commun., 183, 506-519, https://doi.org/10.1016/j.cpc.2011.11.010.

Keranen, R., and V. Chandrasekar, 2014: Detection and estimation of radar reflectivity from weak echo precipitation in dualpolarized weather radars. J. Atmos. Oceanic Technol., 31, 1677-1693, https://doi.org/10.1175/JTECH-D-13-00155.1.

Lee, J.-S., A. R. Miller, and K. W. Hoppel, 1994: Statistics of phase difference and product magnitude of multi-look processed Gaussian signals. Waves Random Media, 4, 307-319, https:// doi.org/10.1088/0959-7174/4/3/006.

Lei, L., G. Zhang, R. J. Doviak, R. Palmer, B. L. Cheong, M. Xue, Q. Cao, and Y. Li, 2012: Multilag correlation estimators for polarimetric radar measurements in the presence of noise. J. Atmos. Oceanic Technol., 29, 772-795, https://doi.org/ 10.1175/JTECH-D-11-00010.1.

McDonough, R. N., and A. D. Whalen, 1995: Detection of Signals in Noise. 2nd ed. Academic Press, 495 pp.

Mead, J. B., 2016: Comparison of meteorological radar signal detectability with noncoherent and spectral-based processing. 
J. Atmos. Oceanic Technol., 33, 723-739, https://doi.org/ 10.1175/JTECH-D-14-00198.1.

Papoulis, A., 1984: Probability, Random Variables, and Stochastic Processes. 2nd ed. McGraw-Hill, Inc., 576 pp.

Seliga, T. A., and V. N. Bringi, 1976: Potential use of radar differential reflectivity measurements at orthogonal polarizations for measuring precipitation. J. Appl. Meteor., 15, 69-75, https://doi.org/ 10.1175/1520-0450(1976)015<0069:PUORDR > 2.0.CO;2.

Siggia, A. D., and R. E. Passarelli Jr., 2004: Gaussian model adaptive processing (GMAP) for improved ground clutter cancellation and moment calculation. Proceedings of the Third European Conference on Radar in Meteorology and Hydrology (ERAD) together with the COST 717 Final Seminar, Copernicus GmbH, 67-73.

Skolik, M. I., 1990: Radar Handbook. 2nd ed. McGraw-Hill, $1163 \mathrm{pp}$

Ulaby, F. T., R. K. Moore, and A. K. Fung, 1982: Radar Remote Sensing and Surface Scattering and Emission Theory. Vol. II,
Microwave Remote Sensing: Active and Passive, AddisionWesley Publishing Co., 634 pp.

Urkowitz, H., and J. D. Nespor, 1992: Obtaining spectral moments by discrete Fourier transform with noise removal in radar meteorology. IGARSS '92: International Geoscience and Remote Sensing Symposium; International Space Year: Space Remote Sensing, R. Williamson and T. Stein, Eds., Vol. I, IEEE, 12-14, https://doi.org/ 10.1109/ IGARSS.1992.576614.

Wolfram, 2012a: Chi-square distribution. Wolfram MathWorld, http:// mathworld.wolfram.com/Chi-SquaredDistribution.html.

, 2012b: Normal product distribution. Wolfram Mathworld, http:// mathworld.wolfram.com/NormalProductDistribution.html.

Zrnić, D. S., 1996: Simultaneous differential polymetric measurement and co-polar correlation coefficient measurement. U.S. Patent 5,500,646, filed 29 July 1994, and issued 19 March 1996. 